\title{
Calcium signaling through a Transient Receptor Channel is important for Toxoplasma gondii growth
}

\author{
Karla M. Márquez-Nogueras ${ }^{1,2}$, Nathan M. Chasen ${ }^{1}$, Myriam A. Hortua Triana ${ }^{1}$, Ivana Y. Kuo ${ }^{3}$, \\ Silvia N.J. Moreno ${ }^{1,4 *}$ \\ ${ }^{1}$ Center for Tropical and Emerging Global Diseases, ${ }^{2}$ Department of Microbiology, and \\ ${ }^{3}$ Department of Cell and Molecular Physiology, Stritch School of Medicine, Loyola University \\ Chicago, Maywood IL, 60132 \\ ${ }^{4}$ Department of Cellular Biology, University of Georgia, Athens, Georgia 30602
}

Running title: TRPPL-2 mediates calcium influx in T. gondii

*To whom correspondence should be addressed: Silvia N. J. Moreno, Department of Cellular Biology and Center for Tropical and Emerging Global Disease, 350A Paul D. Coverdell Center, University of Georgia, Athens, GA 30602. Tel.: 706-542-4736; Fax: 706-542-9493; E-mail: smoreno@uga.edu

Keywords: Calcium signaling, TRP channel, Toxoplasma gondii 


\begin{abstract}
Transient Receptor Potential (TRP) channels participate in ion calcium $\left(\mathrm{Ca}^{2+}\right)$ influx and intracellular $\mathrm{Ca}^{2+}$ release. TRP channels have not been studied in Toxoplasma gondii or any other Apicomplexan parasite. We characterized a protein predicted to possess a TRP domain (TgTRPPL-2) and determined its role in $\mathrm{Ca}^{2+}$ signaling in $T$. gondii, the causative agent of toxoplasmosis. TgTRPPL-2 localized to the plasma membrane and the endoplasmic reticulum of T. gondii. The $\triangle T$ TRTRPL-2 mutant was defective in growth and $\mathrm{Ca}^{2+}$ influx. Heterologous expression of TgTRPPL-2 in HEK-3KO cells allowed its functional characterization. Patching of ER-nuclear membranes demonstrated that TgTRPPL-2 is a non-selective cation channel that conducts $\mathrm{Ca}^{2+}$. Pharmacological blockers of TgTRPPL-2 inhibited $\mathrm{Ca}^{2+}$ influx and parasite growth. This is the first report of an Apicomplexan channel that conducts $\mathrm{Ca}^{2+}$ and initiates the $\mathrm{Ca}^{2+}$ signaling cascade that culminates in the stimulation of motility, invasion and egress. TgTRPPL-2 is a potential target for combating toxoplasmosis.
\end{abstract}

\title{
INTRODUCTION
}

$\mathrm{Ca}^{2+}$ signaling is universal and forms part of the signaling pathways that activate or modulate a variety of physiological responses such as gene transcription, muscle contraction, cell differentiation and proliferation [1]. $\mathrm{Ca}^{2+}$ signals can be generated through the opening of ion channels that allow the downward flow of $\mathrm{Ca}^{2+}$ from either outside the cell or from intracellular stores like the endoplasmic reticulum [2].

Toxoplasma gondii is an intracellular parasite from the Apicomplexan phylum, that causes toxoplasmosis in humans [3]. In immunocompromised individuals, infection with $T$. gondii may lead to severe complications like encephalitis, myocarditis and death [4]. The T. gondii tachyzoite engages in a lytic cycle directly responsible for the pathogenicity of the infection as it results in lysis of host cells [5]. The lytic cycle consists of active invasion of host cells, replication inside a parasitophorous vacuole, followed by egress to search for a new host cell to invade. $\mathrm{Ca}^{2+}$ signals resulting from $\mathrm{Ca}^{2+}$ influx or from intracellular release, trigger a signaling cascade in the parasite that culminates in the stimulation of essential features of its lytic cycle, like motility, invasion, egress and secretion of proteins essential for attachment to the host cell $[6,7]$.

Previously we demonstrated the presence of a $\mathrm{Ca}^{2+}$ influx activity at the plasma membrane of T. gondii tachyzoites that was functional in extracellular tachyzoites [8] and intracellular replicating parasites [9]. The application of voltage operated $\mathrm{Ca}^{2+}$ channel blockers such as nifedipine inhibited $\sim 80 \%$ of $\mathrm{Ca}^{2+}$ influx, and the residual $\mathrm{Ca}^{2+}$ entry activity suggested the potential existence of more than one channel at the plasma membrane of T. gondii [8]. The molecular entity of these channels has remained elusive.

Transient Receptor Potential (TRP) channels are a large family of cation permeable channels grouped into seven subfamilies based upon their gene sequence [10]. TRP channels can be activated by a multitude of stimuli and are involved in a wide range of cellular functions [11]. Most TRP channels are permeable to $\mathrm{Ca}^{2+}$ and all of them are permeable to monovalent cations 
[11]. Some TRP channels can participate in $\mathrm{Ca}^{2+}$ influx as well as $\mathrm{Ca}^{2+}$ release from intracellular stores $[12,13]$. Mutations in these molecules are associated with a diverse set of diseases, due to their wide distribution in various tissues and their roles in pathological conditions, like cancer and renal diseases, making these channels important therapeutic targets [14]. The polycystin TRP (TRPP) subfamily of proteins are implicated in Autosomal Dominant Polycystic Kidney Disease (ADPKD) [15].

Predicted protein sequences with TRP domains have been found in most parasitic protozoa, although in lower numbers and types than in other organisms [16]. This could be the result of the evolutionary distance between the species studied, or because of loss of specific functions resulting from evolution of the parasitic lifestyle [16]. A genome analysis of a number of pathogenic protozoan parasites [17] searching for genes with homology to mammalian $\mathrm{Ca}^{2+}$ channels identified two $T$. gondii hypothetical genes (TgGT1_247370 and TgGT1_310560) with homologous regions to the TRPP family [18]. We termed these genes TgTRPPL-1 and TgTRPPL2 (TRPP Like) respectively. Previous work from our laboratory, localized the gene product of $\operatorname{TgTRPPL}-1$ to the ER with high-affinity tags due to its low level of expression [19].

In the current study we characterize TgTRPPL-2 in T. gondii, which represents the first TRP cation channel studied in any Apicomplexan parasite. Using reverse genetic approaches, we determine the role of TgTRPPL-2 in the lytic cycle of $T$. gondii. We also characterize the electrophysiological features of TgTRPPL-2 and its role in $\mathrm{Ca}^{2+}$ influx and, interestingly, find that pharmacological agents that block the activity of TgTRPPL-2 also inhibit $\mathrm{Ca}^{2+}$ influx in the parasite and parasite growth. TgTRPPL-2 emerges as one of the molecular entities involved in initiating $\mathrm{Ca}^{2+}$ signals in T. gondii.

\section{RESULTS}

\section{TgTRPPL-2 localizes to the plasma membrane and the endoplasmic reticulum}

Hypothetical proteins encoded by two genes in the $T$. gondii's genome are annotated as proteins possessing Polycystic Kidney Disease (PKD) domains, which are characteristic of the subfamily P, or polycystin, TRP channels. Mammalian TRP-P channels contain 6 transmembrane domains with an extracellular loop between the first and second transmembrane domain [20]. We termed the $T$. gondii proteins TgTRPPL-1 and TgTRPPL-2. BLAST analysis to compare the sequences of the mammalian PKD2 and TgTRPPL-2 revealed low sequence homology (21.7\%), even within the PKD Domains. The $T g G T 1 \_310560$ gene predicts the expression of a protein of 2,191 amino acids with an apparent molecular weight of $237 \mathrm{kDa}$ and 14 transmembrane domains. The predicted topology [21] shows an extracellular loop between the first and second transmembrane domain, which is characteristic of PKD channels (Fig. 1A, TgGT1_310560 cartoon). While our BLAST analysis showed low sequence homology, HHPred, which searches for homologs based on protein sequence and secondary structure [22] revealed that TgTRPPL-2 shows significant homology with mammalian PKD2 (Table S1).

To investigate the localization of TgTRPPL-2, we introduced the high-affinity tag smHA [19] at the 3' terminus of the TgTRPPL-2 locus and isolated TgTRPPL-2-smHA cell clones. Carboxy- 


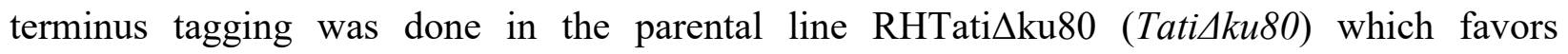
homologous recombination [23]. Correct incorporation of the tag in the TgTRPPL-2-smHA line was validated by PCR (Fig. S1A) and western blot analysis using anti-HA antibodies (Fig. 1B). A band of approximately $\sim 150 \mathrm{kDa}$ was observed in lysates of TgTRPPL-2-smHA tachyzoites, which is nearly $87 \mathrm{kDa}$ smaller (Fig. 1B) than the predicted size of $237 \mathrm{kDa}$ without taking into account the smHA tag $(\sim 39 \mathrm{kDa})$.

Interestingly, a recent release of ToxoDBv.45 presents additional models for the $T g G T 1 \_310560$ gene from different $T$.gondii strains. The gene model for TgVAND_310560 shows two fragments, $T g V A N D \_310560 A$, which predicts a protein with 9 TMD and a size of $~ 107.97$ kDa and TgVAND_310560B, which predicts a protein with 6 TMD and a size of $\sim 116.6 \mathrm{kDa}$. Sequence alignments of the $T g G T 1 \_310560$ gene with the gene models for the VAND strain (A and B) shows $98 \%$ homology between them (Fig. 1C). The T. gondii VAND strain is an isolate from South America and belongs to the hypervirulent Type I group, as the GT1 and RH strains. According to the gene model of the VAND strain, the predicted MW of the TgVAND_310560B protein would be similar to the band size observed in our western blots $(116.6+39)$ with a predicted topology of 6 TMD, in closer agreement with typical TRP channels [24, 25].

To further demonstrate that the protein band observed in the western blot analysis corresponded to the tagged TgTRPPL-2 gene, we performed immunoprecipitations with anti-HA of lysates from the TgTRPPL-2-smHA cells. The immunoprecipitated samples were run in a PAGE gel. The $\sim 150 \mathrm{kDa}$ band was excised and analyzed by mass spectrometry (Fig. S1B, red box)(Table S2). Approximately $\sim 700$ amino acids toward the C-terminus domain of the fusion protein were covered by the peptides, corresponding to a $66 \%$ coverage. Comparison of the coverage with the TgVAND_310560B predicted protein, revealed that approximately $66 \%$ of the sequence was recovered by the mass spectrometry analysis. This result indicates that the TgGT1_310560 is likely cleaved, a characteristic in common with other TRP channels [26].

We next investigated the cellular localization of TgTRPPL-2. Immunofluorescence analysis (IFAs) of extracellular and intracellular parasites showed that TgTRPPL-2-smHA apparently localizes to peripheral vesicles close to the plasma membrane (PM) and to the endoplasmic reticulum (ER) (Fig. 1D-E). Some co-localization with the plasma membrane surface antigen (Sag1) and the sarco-endoplasmic reticulum $\mathrm{Ca}^{2+}$-ATPase (TgSERCA) (ER marker) was observed (Fig. 1D-E). However, considering the low-level of expression of TgTRPPL-2, it was difficult to draw definitive conclusions about its localization.

We next produced polyclonal antibodies against a fragment peptide of TgTRPPL-2, which is indicated in Fig. 1A (highlighted in green). The peptide was expressed in bacteria, purified and used for immunization of mice. Mouse serum was isolated and affinity purified prior to its use for IFA. The localization at the periphery of extracellular tachyzoites was further confirmed by colocalization with $\alpha$ SAG1 (Fig. 1F). Extracellular tachyzoites showed intracellular staining that colocalized with TgSERCA (Fig. 1F) supporting ER localization. Additionally, IFA of intracellular tachyzoites showed that TgTRPPL-2 co-localizes with $\alpha$ SAG1 and $\alpha$ SERCA (Fig.1G). 
In summary, TgTRPPL-2 is expressed in T. gondii tachyzoites, it is likely post-translationally cleaved, and localizes to the PM and the ER.

\section{TgTRPPL-2 is important for growth, invasion and egress of Toxoplasma gondii}

With the aim of investigating the physiological role of TgTRPPL-2 in T. gondii, we generated a $\triangle T g T R P P L-2$ mutant using the CRISPR-Cas9 approach to disrupt the transcription of TgTRPPL2 by inserting a dihydrofolate reductase-thymidylate synthase (DHFR) cassette in the TgTRPPL2 genomic locus (Fig. 2A). Genetic controls for the insertion were done by PCR, and qPCR showed a significant decrease in the levels of TgTRPPL-2 transcripts (Fig. 2B).

The $\triangle T$ TRRPPL-2 mutant was complemented with Cosmid PSBLZ13 [27] that contains the whole genomic locus of the TgTRPPL-2 gene to generate the cell line $\triangle T g T R P P L-2-t r p p l 2$. Controls for the expression of TgTRPPL-2 were done by qPCR and by IFAs, which further confirmed the identity of the tagged protein, as it was not expressed in the $\triangle T$ TRTPPL-2 mutant and was present in the complemented line $\triangle T g T R P P L-2$-trppl2 (Fig. 2B-C). Further validation of the absence of expression of TgTRPP2-L and its complementation is shown in Figs S2A-B with additional IFA images and western blots of $\triangle T g T R P P L-2$ and $\triangle T g T R P P L$-2-trppl2 parasites (Fig $\mathrm{S} 2 \mathrm{~B})$.

We next evaluated if the expression of $T g T R P P L-2$ would impact $T$. gondii growth by plaque assays, in which the parasite engages in repetitive cycles of invasion, replication, and egress causing host cell lysis and formation of plaques observed as white spots by staining with crystal violet. The $\triangle T$ TRRPPL-2 mutant formed smaller plaques compared to its parental counterpart indicating a growth defect (Fig. 2D). This growth defect was partially restored in the complemented cell line (Fig. 2D). We reasoned that the overexpression of TgTRPPL-2 in the $\triangle T$ TRRPL-2-trppl2 mutant may affect parasite fitness masking the rescue effect.

To determine which step of the lytic cycle was affected we performed invasion and egress assays. For invasion we used the red-green assay [28] under two extracellular $\mathrm{Ca}^{2+}$ concentrations (1.8 and $0.5 \mathrm{mM})$. Quantification of invasion in the presence of $1.8 \mathrm{mM} \mathrm{Ca}^{2+}$ showed a lower invasion rate for the $\triangle T g T R P P L-2$ (Fig. 2E). Reducing the extracellular concentration of $\mathrm{Ca}^{2+}$ to $0.5 \mathrm{mM}$ resulted in a reduced rate of invasion by the parental line, which was similar to the invasion rate of the $\triangle T g T R P P L-2$ mutant. This result demonstrated that TgTRPPL-2 is important for invasion at higher concentrations of extracellular $\mathrm{Ca}^{2+}$.

Egress of intracellular tachyzoites can be triggered by permeabilizing infected host cells with saponin in the presence of a buffer containing $1.8 \mathrm{mM}$ of extracellular $\mathrm{Ca}^{2+}$. Under these conditions egress of the $\triangle T$ TRRPPL-2 mutant was slower than egress of the parental strain (Fig. 2F). Additionally, when egress was stimulated by Zaprinast, which increases cytosolic $\mathrm{Ca}^{2+}$, the $\triangle T g T R P P L-2$ mutant also took longer to egress (Fig. 2G). For both assays tested, the $\triangle T g T R P P L-$ 2 mutant took twice as long as the parental line.

In summary, disruption of the TgTRPPL-2 locus negatively impacted two important steps of the $T$. gondii lytic cycle, invasion and egress, which impacted parasite growth. 


\section{The role TgTRPPL-2 in $\mathrm{Ca}^{2+}$ influx}

We previously showed that $T$. gondii tachyzoites allow influx of $\mathrm{Ca}^{2+}$ when exposed to $2 \mathrm{mM}$ extracellular $\mathrm{Ca}^{2+}$ [8]. To determine the role of TgTRPPL-2 in this pathway, we loaded $\triangle T g T R P P L-2$ parasites with Fura-2AM to study intracellular $\mathrm{Ca}^{2+}$ changes after exposing them to $1.8 \mathrm{mM}$ extracellular $\mathrm{Ca}^{2+}$ (Fig. 3A). The resting cytosolic $\mathrm{Ca}^{2+}$ concentration of $\triangle T g T R P P L-2$ mutant was around $75 \mathrm{nM}$ which is similar to the resting concentration of parental cells $(\sim 70-100$ $\mathrm{nM})$. Adding $1.8 \mathrm{mM} \mathrm{Ca}^{2+}$ to the extracellular buffer caused an increase in cytosolic $\mathrm{Ca}^{2+}$ in both the parental strain and the $\triangle T g T R P P L-2$ mutant (Fig. 3B). However, $\mathrm{Ca}^{2+}$ influx of the $\triangle T g T R P P L$ 2 mutant was significantly lower (Fig. 3C) and was decreased by almost 50\%. The $\triangle T g T R P P L-2$ trppl 2 complemented mutant, however, regained the $\mathrm{Ca}^{2+}$ influx activity and it even showed higher $\mathrm{Ca}^{2+}$ influx than parental cells, consistent with the higher expression of TgTRPPL-2 shown by qPCR (Fig. 2B). The reduction of $\mathrm{Ca}^{2+}$ influx was further confirmed when adding $1 \mathrm{mM}$ extracellular $\mathrm{Ca}^{2+}$ to the $\triangle T$ TRRPPL-2 mutant (Fig. S3A).

When $T$. gondii tachyzoites are suspended in a high $\mathrm{Ca}^{2+}$ buffer from the beginning of the experiment there is a slow constitutive influx of $\mathrm{Ca}^{2+}$, that we attribute to leakage through a PM channel (Fig. 3D, parental black tracing). Interestingly, this leakage activity was significantly reduced in the $\triangle T g T R P P L-2$ mutant (Fig. 3D-E, blue tracing and bar), supporting a role of TgTRPPL-2 in constitutive $\mathrm{Ca}^{2+}$ influx at the PM. Additional evidence is provided by the enhanced $\mathrm{Ca}^{2+}$ leakage observed with the $\triangle T$ TRRPPL-2-trppl2 complemented mutant (Fig. 3D-E, purple tracing and bar). The high $\mathrm{Ca}^{2+}$ leakage and $\mathrm{Ca}^{2+}$ influx observed with the $\triangle T$ TRRPPL-2-trppl2 mutant may affect parasite fitness and would explain the partial growth recovery observed in the complemented mutant.

$\mathrm{Ca}^{2+}$ channels may also be modulated by $\mathrm{Ca}^{2+}$ itself [29]. We previously showed that a cytosolic $\left[\mathrm{Ca}^{2+}\right]$ increase may activate $\mathrm{Ca}^{2+}$ influx at the PM $\left(\mathrm{Ca}^{2+}\right.$ activated-Ca ${ }^{2+}$ entry) [8]. We next investigated if the $\mathrm{Ca}^{2+}$ activated-Ca ${ }^{2+}$ entry (CACE) activity was due to the functioning of TgTRPPL-2 at the PM. We added thapsigargin (thap) to tachyzoites in suspension (Fig. 3F), which results in a cytosolic $\mathrm{Ca}^{2+}$ increase due to inhibition of the SERCA-Ca ${ }^{2+}$-ATPase (SERCA) resulting in uncompensated $\mathrm{Ca}^{2+}$ efflux into the cytosol. This elevated cytosolic $\mathrm{Ca}^{2+}$ stimulates further $\mathrm{Ca}^{2+}$ influx at the PM, which we assessed as an increase in cytosolic $\mathrm{Ca}^{2+}$ following addition of high $\mathrm{Ca}^{2+}$ to the buffer (Fig 3F, black tracing). Note that the $\Delta\left[\mathrm{Ca}^{2+}\right]_{\text {cyt }}$ shown in Fig. $3 \mathrm{H}$, black column, is almost 2.5 times higher than the $\Delta\left[\mathrm{Ca}^{2+}\right]_{\text {cyt }}$ observed without previous addition of thap (Fig. 3C, black column). This CACE activity was absent in the $\triangle T$ TRRPPL-2 mutant (Fig. 3F, blue tracing) but was restored in the $\triangle T$ TRRPPL-2-trppl2 complemented strain (Fig. 3F, purple tracing). Quantifications of the rate of $\mathrm{Ca}^{2+}$ increase after adding $\mathrm{Ca}^{2+}$ and statistical analyses are shown in Fig. 3G-H. Note that the $\triangle T$ TRRPPL-2 mutant showed a reduced response to the addition of thap and also to the addition of $\mathrm{Ca}^{2+}$. Comparing the response to the addition of extracellular $\mathrm{Ca}^{2+}$ shown in Fig. $3 \mathrm{H}$, blue column, the $\Delta\left[\mathrm{Ca}^{2+}\right]_{\text {cyt }}$ is similar to that measured directly without previous addition of thap (compare with the blue column in Fig 3C). This result points to a 
complete absence of the modulatory effect of cytosolic $\mathrm{Ca}^{2+}$ on the $\mathrm{PM} \mathrm{Ca}^{2+}$ influx in the $\triangle T g T R P P L-2$ mutant, which is restored in the complemented mutant, $\triangle T g T R P P L-2$-trppl2.

We next tested Zaprinast, which increases the levels of cGMP resulting in $\mathrm{Ca}^{2+}$ release from an unidentified store [30]. We previously determined that $\mathrm{Ca}^{2+}$ release was almost 2.5 times higher in the presence of extracellular $\mathrm{Ca}^{2+}$, as compared with that in the absence of extracellular $\mathrm{Ca}^{2+}$ [30]. We attribute this increase to stimulation of the $\mathrm{PM} \mathrm{Ca}^{2+}$ channel by cytosolic $\mathrm{Ca}^{2+}(\mathrm{CACE})$. When testing this phenotype with the $\triangle T g T R P P L-2$ mutant, we observed that the increased response was absent (Fig. 3I-K). Also note that even the release of $\mathrm{Ca}^{2+}$ from intracellular stores by Zaprinast in the presence of low extracellular $\mathrm{Ca}^{2+}(\sim 50 \mathrm{nM})$ was significantly decreased in the $\triangle T$ TRRPPL-2 mutant (Fig. S3B-C). The modulatory action of elevated cytosolic $\mathrm{Ca}^{2+}$ in $\mathrm{Ca}^{2}$ influx was absent in the $\triangle T g T R P P L-2$ mutant (Fig. 3I-K).

Taken together, these results suggest a role for TgTRPPL-2 in $\mathrm{Ca}^{2+}$ influx at the PM. In addition, TgTRPPL-2 is modulated by cytosolic $\mathrm{Ca}^{2+}$ and is responsible for a constitutive PM Ca ${ }^{2+}$ influx pathway.

\section{TgTRPPL-2 is a cation conducting channel}

With the aim of establishing that TgTRPPL-2 functions as a channel and is able to conduct $\mathrm{Ca}^{2+}$ we cloned the cDNA of the TgTRPPL-2 gene into a mammalian expression vector (pCDNA3.1) for expression in human embryonic kidney 293 cells (HEK-3KO) [31]. These HEK cell line is genetically modified and the 3 isoforms of the inositol 1,4,5-trisphosphate receptor $\left(\mathrm{IP}_{3} \mathrm{R}\right)$ are deleted, to reduce background $\mathrm{Ca}^{2+}$ currents [31]. TgTRPPL-2 was localized to the ER of HEK cells as assessed by co-localization with a red fluorescent protein (RFP) targeted to the ER and compared with the mammalian homolog polycystin 2 (PC2) (Fig. 4A). Because of this, we isolated nuclear/ER membranes (Fig. 4B) for single channel patch clamp experiments and further characterization of the permeability properties of TgTRPPL-2 (Fig. 4C).

In the presence of $1.8 \mathrm{mM}$ of $\mathrm{Ca}^{2+}$ inside the patch pipette and $100 \mathrm{nM}$ of $\mathrm{Ca}^{2+}$ in the bath solution (see scheme of Fig. 4B), the membranes isolated from control cells, held at $-80 \mathrm{mV}$, showed very little activity and the conductance remained at less than $1.5 \mathrm{pA}$ (Fig. 4D, control tracing). Some channel activity was observed after artificially depolarizing membranes ( -80 to $+20 \mathrm{mV}$ ) presumably due to opening of potassium channels. In comparison, when analyzing membranes isolated from cells expressing TgTRPPL-2, a significant increase in the open probability and current sizes was observed (Fig. 4D, TgTRPPL-2 blue tracing). The currentvoltage relationship was linear and significantly different from the one from control cells (Fig. 4E, blue vs. black line).

We compared the activity of TgTRPPL-2 with that of the mammalian PKD channel PC2 in parallel experiments since PC2 has been well characterized in the literature. Activity of PC2 expressing cells displayed a voltage-dependent behavior, as the current-voltage relationship was not linear, with a conductance of $\sim 73 \mathrm{pS}$ (Fig. 4D-E, red tracing). Previous work has demonstrated that PC2 can be voltage dependent. Additionally, depending on the experimental design, the measured conductance for PC2 can be variable. Comparing our experimental approach to previous 
work, conductance for PC2 in a high $\mathrm{Ca}^{2+}$ solution is similar $(\sim 73 \mathrm{pS}$ vs. $\sim 97 \mathrm{pS})$. Although TgTRPPL-2 does not appear to be voltage dependent, conductance of the channel is similar to that of its mammalian homologue.

$\mathrm{Ca}^{2+}$ is able to modulate the activity of channels like TRP-P channels, some of which have an EF-hand motif at the C-terminus, and have been shown to be activated by $\mathrm{Ca}^{2+}[29,32]$. To determine whether $\mathrm{Ca}^{2+}$ is able to modulate the activity of TgTRPPL- 2 we first varied the [Ca ${ }^{2+}$ ] inside the pipette (in equilibrium with the ER lumen). When the $\mathrm{Ca}^{2+}$ concentration was increased to $10 \mathrm{mM} \mathrm{Ca}^{2+}$ there was a significant inhibition of TgTRPPL-2 channel activity. With high $\mathrm{Ca}^{2+}$ concentration, the channel displayed voltage-dependent inhibition over the -75 to $-25 \mathrm{mV}$ range and conductance was significantly decreased (Fig. 4F). In the presence of $1.8 \mathrm{mM} \mathrm{Ca}^{2+}$ inside the pipette, TgTRPPL-2 had a conductance of $\sim 55 \mathrm{pS}$, which is significantly higher than the conductance calculated for control membranes. The conductance decreased to $\sim 21 \mathrm{pS}$ when $\mathrm{Ca}^{2+}$ was increased to $10 \mathrm{mM}$.

Although no evidence for a conserved EF-hand motif was found in TgTRPPL-2 we checked for the potential modulation by cytosolic $\mathrm{Ca}^{2+}$. Increasing the concentration of $\mathrm{Ca}^{2+}$ in the bath solution from $100 \mathrm{nM}$ to $10 \mu \mathrm{M}$ (which would simulate changes in cytosolic $\mathrm{Ca}^{2+}$ ), enhanced channel activity from membranes expressing TgTRPPL-2 (Fig. 4G-H, blue vs. gold line). Interestingly, increasing the $\left[\mathrm{Ca}^{2+}\right]$ only increased the open probability when the membrane was depolarized to $-80 \mathrm{mV}$ (Fig. $4 \mathrm{I}$ ). However, increase of the $\left[\mathrm{Ca}^{2+}\right]$ of the bath solution, increased the conductance of the channel almost $2.5 \mathrm{x}$, suggesting modulation of the channel by $\mathrm{Ca}^{2+}$ itself. In conclusion, these data indicate that TgTRPPL-2 is able to conduct $\mathrm{Ca}^{2+}$ currents and is modulated by cytosolic $\mathrm{Ca}^{2+}$.

To distinguish whether TgTRPPL-2 is able to conduct cation currents and to determine if the activity measured could be the result of permeation of potassium, we replaced potassium with the non-permeable ion cesium $[33,34]$. In the presence of $1.8 \mathrm{mM} \mathrm{Ca}^{2+}$ inside the pipette, in a cesium chloride solution, membranes from TgTRPPL-2- and PC2-expressing cells have a significantly higher activity than control cells (Fig. 5A). The current-voltage relationship is linear through different applied voltages and significantly different from that of control cells in potassium or cesium chloride solution (Fig. 5B). Although channel conductance is slightly higher in potassium chloride, it is not significantly different than the calculated conductance and open probability obtained in cesium chloride (Fig. 5B-C). However, when applying voltages higher than $-40 \mathrm{mVs}$, the channel was open for longer times in the presence of cesium chloride vs potassium chloride (Fig. 5D, green vs. blue line). These results indicate that TgTRPPL-2 permeates $\mathrm{Ca}^{2+}$, however it can also conduct potassium, since channel conductance is slightly higher in the potassium chloride solution.

We further demonstrated that TgTRPPL-2 is able to conduct $\mathrm{Ca}^{2+}$ by following $\mathrm{Ca}^{2+}$ changes of TgTRPPL-2-HEK-3KO or RFP-ER-HEK-3KO cells co-transfected with a genetic $\mathrm{Ca}^{2+}$ indicator, allowing to follow ER luminal $\mathrm{Ca}^{2+}$ changes and current activity simultaneously. Luminal $\mathrm{Ca}^{2+}$ changes were followed through one cycle of membrane depolarization from $-80 \mathrm{mV}$ to $40 \mathrm{mV}$ (Fig. S4A). The fluorescence of the $\mathrm{Ca}^{2+}$ indicator decreased in the TgTRPPL-2- 
expressing cells with time, as voltage was applied. In both potassium as well as cesium chloride solutions at $1.8 \mathrm{mM} \mathrm{Ca}^{2+}$ we observed that the fluorescence decrease was significantly larger when the HEK-3KO cells expressed TgTRPPL-2 (Fig. S4B-C vs. D-E). The slope for the fluorescence decrease appeared higher in the cesium chloride solution than in the potassium solution, although was quite variable (Fig. S4F-G). In summary, the observed decrease in the fluorescence of the $\mathrm{Ca}^{2+}$ indicator supports the $\mathrm{Ca}^{2+}$ permeation activity of TgTRPPL-2, which agrees with the single channel conductance measurements.

\section{Inhibition of TgTRPPL2 by TRP Channel Inhibitors}

Previously we demonstrated $\mathrm{Ca}^{2+}$ influx in $T$. gondii and its inhibition by L-type voltage gated $\mathrm{Ca}^{2+}$ channel blockers like nifedipine (Fig. 6A) [8]. Taking into account that TgTRPPL-2 is a cation permeable channel and localizes to the PM, we next investigated if the residual $\mathrm{Ca}^{2+}$ influx activity observed with the $\triangle T g T R P P L-2$ mutant could be blocked with nifedipine. Interestingly, pre-incubation of the $\triangle T g T R P P L-2$ parasites with nifedipine showed that the initial cytosolic $\mathrm{Ca}^{2+}$ was elevated and it was around $400 \mathrm{nM}$ (compared to $100 \mathrm{nM}$ of the parental strain under identical conditions) in $\mathrm{Ca}^{2+}$-free buffer (Fig. 6B). Further addition of extracellular $\mathrm{Ca}^{2+}$ did not result in $\mathrm{Ca}^{2+}$ influx and only a slow steady cytosolic $\mathrm{Ca}^{2+}$ increase was observed (Fig. 6B). We attribute the higher cytosolic $\mathrm{Ca}^{2+}$ in the presence of nifedipine to leakage of stored $\mathrm{Ca}^{2+}$ into the cytosol as the cell is trying to compensate for the complete absence of $\mathrm{Ca}^{2+}$ influx. However, the absence of $\mathrm{Ca}^{2+}$ influx after adding extracellular $\mathrm{Ca}^{2+}$ in the presence of nifedipine, points to a role for TgTRPPL-2 in $\mathrm{Ca}^{2+}$ influx.

We next tested the effect of anthranilic acid (ACA), a wide spectrum TRP channel inhibitor [35], on $\mathrm{Ca}^{2+}$ influx of both the parental control and the $\triangle T g T R P P L-2$ mutant (Fig. 6C). ACA inhibited $\mathrm{Ca}^{2+}$ influx by $40-50 \%$ of the parental cell line (Fig. 6C, black vs. red tracing). However, preincubation of $\triangle$ TgTRPPL-2 tachyzoites with ACA, did not further reduce $\mathrm{Ca}^{2+}$ influx (Fig. 6C, dark blue vs. light blue tracings). Sensitivity to both nifedipine and ACA was restored in the complemented cell line (Fig. 6D). These results point to TgTRPPL-2 as a PM channel that conducts $\mathrm{Ca}^{2+}$, and it is relevant for its influx from the extracellular milieu, and is sensitive to TRP channel inhibitors.

\section{The Role of TRPPL-2 as a Ca ${ }^{2+}$ leak channel at the ER membrane}

The dual localization of TgTRPPL-2 at the PM and ER suggests the potential function of the channel at both locations (Fig. 1D). Inhibition of TgSERCA with thap results in $\mathrm{Ca}^{2+}$ efflux into the cytosol [36]. We observed that incubation of tachyzoites of the parental strain with ACA significantly decreased the efflux of $\mathrm{Ca}^{2+}$ caused by thap (Fig. $6 \mathrm{E}-\mathrm{F}$, black line and bar vs. red line and bar). The ACA-inhibited $\mathrm{ER} \mathrm{Ca}^{2+}$ efflux rate was comparable to the decreased efflux rate triggered by thap in the $\triangle T$ TRTRPL-2 mutant (Fig. 6F, blue bar versus red bar and Fig. 3D). In addition, $\mathrm{Ca}^{2+}$-activated-Ca ${ }^{2+}$ entry, evaluated by adding $1.8 \mathrm{mM}$ of extracellular $\mathrm{Ca}^{2+} 50 \mathrm{~s}$ after stimulating efflux with thap, was inhibited by ACA (Fig. 6G, green versus gold line). $\mathrm{Ca}^{2+}$ influx after thap was reduced to basal $\mathrm{Ca}^{2+}$ influx (without pre-addition of thap) for both parental and 
$\triangle T g T R P P L-2$ mutant. Note that $\mathrm{Ca}^{2+}$ influx in $\triangle T g T R P P L-2$ tachyzoites after stimulus by thap, and $\mathrm{Ca}^{2+}$ influx in the parental strain without any stimulus are similar because the modulation of $\mathrm{Ca}^{2+}$ influx by cytosolic $\mathrm{Ca}^{2+}$ is lost in the $\triangle T$ TTRPPL-2 parasites (Fig. $6 \mathrm{H}$, black versus blue bars).

In conclusion, ACA inhibited both efflux of $\mathrm{Ca}^{2+}$ from the ER as well as $\mathrm{Ca}^{2+}$-induced- $\mathrm{Ca}^{2+}$ entry. This led us to propose that TgTRPPL-2, in addition to mediate $\mathrm{Ca}^{2+}$ influx at the plasma membrane may also mediate $\mathrm{Ca}^{2+}$ leakage from the ER, a pathway sensitive to the TRP-channel inhibitor ACA.

To further validate the specificity of ACA for the inhibition of TgTRPPL-2, we tested this inhibitor and a second broad spectrum TRP channel inhibitor, benzamil, against single channel conductance. Channel activity of TgTRPPL-2 was significantly decreased by both ACA and benzamil (Fig. 7A-B). ACA diminished the amplitude of the channel by reducing the probability and time that the channel remained open (Fig. 7C, E). Conductance of the channel was reduced to almost half in the presence of ACA (Fig. 7B), which correlates with the inhibition of $\mathrm{Ca}^{2+}$ entry in T. gondii. In comparison to ACA, benzamil only reduced the open probability of TgTRPPL-2 but not the length of time the channel was open (Fig. 7D-E)). The conductance of TgTRPPL-2 was reduced to one third of the control in the presence of benzamil (Fig. 7B).

Most interesting, we tested both inhibitors, ACA and benzamil in in vitro growth assays (Fig. 7F-G, top panel and parental bars) and found that they both inhibited in vitro T. gondii growth. We calculated the $\mathrm{IC}_{50}$ for ACA at $1.4 \pm 0.4 \mu \mathrm{M}$. Interestingly, neither ACA nor benzamil affected the growth of the $\triangle T g T R P P L-2$ mutant (Fig. 7G). Note that these cells already grow at a slower rate, which did not change in the presence of the inhibitors. However, cilnidipine, a voltage-gated $\mathrm{Ca}^{2+}$ channel blocker, completely inhibited growth of both parental and $\triangle T g T R P P L-2$ mutant (Fig. $7 \mathrm{G})$.

In conclusion, TgTRPPL-2 is a cation permeable channel that can be inhibited by broad spectrum TRP channel inhibitors. Inhibition of channel activity affects parasite growth.

\section{DISCUSSION}

In this study we report the presence and functional role of a $T$. gondii channel, TgTRPPL-2, that localizes to the plasma membrane and the endoplasmic reticulum. The corresponding gene $T g G T 1 \_310560$, was annotated as hypothetical but was predicted as a transient receptor potential channel based on a bioinformatic analysis of the $T$. gondii genome comparing it with TRP channel genes of mammalian origin [17]. Here, we established that TgTRPPL-2 is important for both $\mathrm{Ca}^{2+}$ influx at the PM and $\mathrm{Ca}^{2+}$ efflux from the ER of $T$. gondii tachyzoites. TgTRPPL-2, expressed in HEK-3KO cells, conducted currents in high $\mathrm{Ca}^{2+}$ solutions and was not voltage dependent. Interestingly, $\mathrm{Ca}^{2+}$ itself modulated the conductance of TgTRPPL-2. Broad spectrum TRP channel inhibitors like ACA and benzamil, inhibited the activity of TgTRPPL-2, $\mathrm{Ca}^{2+}$ influx in the parasite, as well as parasite growth.

Silencing of TgTRPPL-2 in the $\triangle T g T R P P L-2$ mutant impacted both invasion and egress of $T$. gondii, resulting in a general growth defect. Extracellular tachyzoites, which are surrounded by 
high $\mathrm{Ca}^{2+}$, are able to use $\mathrm{Ca}^{2+}$ influx to stimulate invasion of a new host cell and carry on their lytic cycle. The $\triangle T g T R P P L-2$ mutant showed a reduction in their host invasion ability suggesting the defect may be due to a reduction in $\mathrm{Ca}^{2+}$ influx because of the absence of TgTRPPL-2. Interestingly, the reduction in $\mathrm{Ca}^{2+}$ influx $(\sim 50 \%)$ in the $\triangle T g T R P P L-2$ mutant was comparable to the reduction of invasion, suggesting that TgTRPPL-2 is involved in the $\mathrm{Ca}^{2+}$ influx pathway that stimulates invasion. Delay in the ability of the $\triangle T g T R P P L-2$ mutant to egress could be caused by a defective efflux of $\mathrm{Ca}^{2+}$ from the ER, which was significantly lower in the mutant. This is evidence for the function of TgTRPPL2 as a $\mathrm{Ca}^{2+}$ channel at the ER membrane.

The impact of silencing TgTRPPL-2 on $T$. gondii growth, was not total and parasites still were able to perform lytic cycle activities at a reduced rate. The main defects of the $\triangle T g T R P P L-2$ mutant: invasion, egress, $\mathrm{Ca}^{2+}$ influx and $\mathrm{ER} \mathrm{Ca}^{2+}$ efflux was not complete, likely because more than one mechanism or channel is functional at both locations (PM and ER). We hypothesize the presence of another channel at the PM, likely the one responsible for the $\mathrm{Ca}^{2+}$ influx activity that is inhibited by nifedipine [8]. It is also possible that a release channel responsive to $\mathrm{IP}_{3}$ may be involved in release of $\mathrm{Ca}^{2+}$ from the ER [37] with TgTRPPL-2 having a role in efflux under conditions of $\mathrm{ER} \mathrm{Ca}^{2+}$ overload.

Numerous observations in $T$. gondii have demonstrated that intracellular $\mathrm{Ca}^{2+}$ oscillations in the parasite precede the activation of distinct steps of the lytic cycle [6,7]. Influx of both extracellular and intracellular $\mathrm{Ca}^{2+}$ pools into the parasite cytosol contribute to the activation of downstream signaling pathways decoded into critical biological steps of the parasite lytic cycle [7, 38]. $\mathrm{Ca}^{2+}$ influx at the plasma membrane of $T$. gondii is highly regulated, stimulated by cytosolic $\mathrm{Ca}^{2+}$ and is operational in extracellular [8] and intracellular replicating tachyzoites [9]. Our data from the $\triangle T g T R P P L-2$ cells identified TgTRPPL-2 as a functional protein at the plasma membrane and the ER. In these locations it would allow $\mathrm{Ca}^{2+}$ influx into the cytosol. The dual localization of TgTRPPL-2 is in accord with other TRP channels in other cells, which showed a dynamic localization between vesicular organelles and the plasma membrane where they facilitate $\mathrm{Ca}^{2+}$ influx [39]. In this regard, the mammalian ortholog, PC2, localizes to both the plasma membrane and the ER [40].

T. gondii expresses a SERCA-Ca ${ }^{2+}$-ATPase, a P-type ATPase, that couples ATP hydrolysis to the transport of ions across biological membranes (TgSERCA) and localizes to the ER [41]. TgSERCA is sensitive to thapsigargin (thap), a sesquiterpene lactone derived from the plant Thapsia garganica [42, 43]. Previous studies showed that inhibition of TgSERCA by thap resulted in cytosolic $\mathrm{Ca}^{2+}$ efflux through an unknown channel $[8,36]$. In mammalian cells, the passive $\mathrm{Ca}^{2+}$ efflux from the ER is thought to prevent $\mathrm{ER} \mathrm{Ca}^{2+}$ overload and help to maintain the steady-state concentration of luminal $\mathrm{Ca}^{2+}$ permitting cytosolic $\mathrm{Ca}^{2+}$ signaling [2, 44]. Several membrane proteins have been proposed to be involved in the $\mathrm{ER} \mathrm{Ca}^{2+}$ efflux/leak pathway including TRP channels [45]. Results from this work support a role for TgTRPPL-2 in ER Ca ${ }^{2+}$ leakage in $T$. gondii. $\mathrm{Ca}^{2+}$ efflux from the ER observed after adding thap or Zaprinast was also significantly decreased in the $\triangle T g T R P P L-2$ mutant. These results support a functional role for TgTRPPL-2 at the membrane of the ER as the constitutive leak channel involved in $\mathrm{Ca}^{2+}$ efflux when the store is 
filled. This could also be the mechanism by which the ER supplies $\mathrm{Ca}^{2+}$ to other organelles like the mitochondria or the plant-like vacuole (PLV) a lysosome-like compartment [46].

Previous work from our laboratory showed that $\mathrm{Ca}^{2+}$ influx at the plasma membrane did not operate as store-operated $\mathrm{Ca}^{2+}$ entry (SOCE) which was shown with experiments testing surrogate ions like $\mathrm{Mn}^{2+}$ [8]. This result was supported by the lack of components of the SOCE pathway, STIM and ORAI, in the T. gondii genome [17]. However, $\mathrm{Ca}^{2+}$ influx was modulated by cytosolic $\mathrm{Ca}^{2+}$ [8] and this modulation was absent in the $\triangle T g T R P P L-2$ mutant supporting a role for TgTRPPL-2 as the channel responsible for $\mathrm{Ca}^{2+}$ influx at the PM activated by cytosolic $\mathrm{Ca}^{2+}$. TRP channels have been shown to play a role in $\mathrm{Ca}^{2+}$-activated-Ca ${ }^{2+}$ entry [47]. Release of $\mathrm{Ca}^{2+}$ from intracellular stores like the ER, is also significantly diminished in the $\triangle T g T R P P L-2$ mutant, which could affect the stimulation of $\mathrm{Ca}^{2+}$ influx. However, when using Zaprinast, which raised cytosolic $\mathrm{Ca}^{2+}$ at a much higher level than thap, the stimulation of $\mathrm{Ca}^{2+}$ influx by cytosolic $\mathrm{Ca}^{2+}$ was absent. This further supports that TgTRPPL-2 functions at the PM mediating $\mathrm{Ca}^{2+}$ influx and is modulated by cytosolic $\mathrm{Ca}^{2+}$.

We showed that TgTRPPL-2 was able to conduct currents with conductance values comparable to the values of mammalian TRP channels [48-50]. Previous work with PC2, showed that $\mathrm{Ca}^{2+}$ modulated the activity of PC2 [51-53] [48, 54]. Sustained cytosolic $\mathrm{Ca}^{2+}$ increase inhibited PC2 currents [53] while other studies showed that cytosolic $\mathrm{Ca}^{2+}$ increase from physiological $(100 \mathrm{nM})$ to $\mu \mathrm{M}$ levels increased the activity of the channel $[48,54]$. We observed some of these responses with TgTRPPL-2, as increasing $\mathrm{Ca}^{2+}$ inside the pipette (ER luminal) showed a significant decrease in the currents. Comparably, increasing $\mathrm{Ca}^{2+}$ concentration in the bath solution (cytosolic) from physiological levels to $\mu \mathrm{M}$ levels showed an increase of $2.5 \mathrm{x}$ in the conductance of TgTRPPL-2. Although cytosolic $\left[\mathrm{Ca}^{2+}\right]$ is unlikely to reach those high $\mu \mathrm{M}$ levels, the potential presence of $\mathrm{Ca}^{2+}$ microdomains at the plasma membrane or the ER membrane would result in higher concentrations of $\mathrm{Ca}^{2+}$ at the exit of the channel due to slow diffusion of $\mathrm{Ca}^{2+}$ ions [55-57].

Because PKD channels are cation permeable they could also permeate $\mathrm{Na}^{+}$or $\mathrm{K}^{+}$. In the case of TgTRPPL-2 we showed that it can mediate $\mathrm{Ca}^{2+}$ transport in the absence of other ions in the solution. We did not determine the ionic selectivity of TgTRPPL-2, and we can only propose that TgTRPPL-2 is a cation permeable channel. In cilia, PKD channels have been described to have relatively high conductance $[48,58]$. The conductance calculated for TgTRPPL-2 is within range of what has been described for PC2 in other cells (30-157 pS). However, it is important to note that the properties described for any channel will depend on the experimental approaches used.

Anthranilic acid and benzamil are broad spectrum inhibitors that have the ability to inhibit TRP channel activity. ACA is a weak base that inhibits currents mediated by TRP channels. ACA does not block the pore of the channel as most inhibitors but rather reduce the open probability of the channel. In a similar manner, benzamil is also able to inhibit currents mediated by TRP channels by binding to a site that modulates their activity rather than blocking its pore. In our experiments testing ACA and benzamil we observed that while the inhibitors affected $\mathrm{Ca}^{2+}$ influx and growth of the parental cell line, neither affected the already reduced growth and $\mathrm{Ca}^{2+}$ influx of the 
$\triangle T$ TRPPL-2 mutant. This result combined with the inhibition of TgTRPPL-2 currents impacting both open probability and time that the channel remained open points to TgTRPPL-2 as the target of ACA and benzamil.

Recent studies on $\mathrm{Ca}^{2+}$ signaling in $T$. gondii have expanded our understanding of the link between $\mathrm{Ca}^{2+}$ and critical facets of parasite biology (i.e., gliding motility, microneme secretion, host cell invasion and egress). However, important molecular players have remained enigmatic, like the PM channels responsible for $\mathrm{Ca}^{2+}$ influx and the ER channel responsible for the passive leakage into the cytosol. Characterization of TgTRPPL-2 and its function at the ER and PM fills a small gap in our knowledge of $\mathrm{Ca}^{2+}$ signaling and homeostasis in T. gondii (Fig. 8). This study is the first biophysical characterization of a channel in T. gondii (and any Apicomplexan parasite) and TgTRPPL-2 represents the first identified molecule to mediate $\mathrm{Ca}^{2+}$ influx into the cytosol of T. gondii at the plasma membrane and the ER. In addition, this study identifies TgTRPPL-2 as a potential target for combatting Toxoplasmosis.

\section{EXPERIMENTAL PROCEDURES}

\section{Toxoplasma growth}

All parasite strains were continuously maintained in vitro by serial passage in Dulbecco's modified minimal essential media (DMEM) with $1 \%$ FBS, $2.5 \mu \mathrm{g} / \mathrm{ml}$ amphotericin B, $100 \mu \mathrm{g} / \mathrm{ml}$ streptomycin in the human telomerase reverse transcriptase immortalized foreskin fibroblasts (hTERT) [59].

\section{Generation of mutants}

The smHA-LIC-CAT plasmid was used for in situ C-terminal tagging of TgTRPPL-2-smHA [19]. Carboxy-terminus tagging was done in the parental line RHTati $\Delta \mathrm{ku} 80$ (Tati $\Delta \mathrm{ku} 80$ ) [23] a parasite line that contains the tetracycline-regulated transactivator system that allows conditional expression of genes [60] and also in which the $k u 80$ gene was deleted increasing efficiency of homologues recombination [61]. Briefly, a homology region of 974 bp covering the 3' region of the gene of interest excluding the STOP codon was amplified by PCR using $T$. gondii RH genomic DNA as template and cloned into the plasmid. Plasmids were validated by restriction digest and sequencing. The oligonucleotides primers used for PCR and for creating the gene-tagging plasmids and for PCR validations are listed in Table S3 (Primers T1-T3). Prior to transfection all plasmids were linearized within the region of homology. Approximately $20 \mu \mathrm{g}$ of plasmid DNA was used for transfection of $1 \times 10^{7} \mathrm{~T}$. gondii RHTati $\Delta \mathrm{Ku} 80$ parasites using a Gene Pulser X Cell electroporator (BioRad). Selection was done with $20 \mu \mathrm{M}$ chloramphenicol, and clones were isolated by limiting dilution. DNA of selected clones were isolated and screened by PCR.

To disrupt the TgTRPPL-2 (TgGT1_310560) gene a single guide RNA against TgTRPPL2 was constructed as described [62]. The single guide RNA was mutagenized with the desired sequence in a plasmid that contains the Cas9 using the Q5 Mutagenesis Kit following 
manufacturer's instructions. The correct mutation was verified by sequencing. The pyrimethamine-resistant DHFR cassette was amplified by PCR with primers containing 50 bp homology arms of the region upstream and downstream of the start and stop codon of the TgTRPPL-2 gene. The created sgTgTRPPL-2 CRISPR plasmid was co-transfected with the DHFR cassette (3:1 respectively) into RH tachyzoites. Selection followed with pyrimethamine for 7 days. Parasites were sub-cloned by limiting dilution and screening for clones was done by PCR. The primers used for the creation of the $\triangle$ TgTRPPL-2 are listed in Table S3 (Primer K1-K4).

Quantitative PCR. Total RNA from parental, $\triangle T g T R P P L-2$ and $\triangle T$ TTRPPL2-trppl2 parasites was extracted and reversed-transcribed into cDNA. The qPCR reaction was done using the $\mathrm{iQ}^{\mathrm{TM}} \mathrm{SYBR}$ Green master mix (BioRad), plus primers, and the reverse-transcribed cDNA (Primers shown in Table S3, Q1-Q2). The qRT-PCR was carried out on a CFX96 ${ }^{\mathrm{TM}}$ PCR Real-Time detection system (C1000Touch $^{\mathrm{TM}}$ Thermal cycler, BioRad). Relative quantification software (CFX Maestro ${ }^{\mathrm{TM}}$ software) was used for the analysis and relative expression levels were calculated as the fold change using the formula $2^{\Delta \Delta} \mathrm{CT}$ [63]. Normalization was done using actin and tubulin primers. Experiments were repeated three times with triplicate samples.

Antibody production of TgTRPPL-2. The antigenic region for TgTRPPL-2 chosen for antibody production was identified using the IEDB suite of antigenicity prediction software. The DNA sequence was amplified from RH genomic DNA and cloned into the pET-32 LIC/EK vector (Novagen), which adds an N-terminal thioredoxin and histidine tag to the expressed protein. Recombinant CP1Ag was expressed and initially purified via a nickel-affinity column (HisPur Thermo Fisher) as previously described [64]. Cleavage of the N-terminal thioredoxin and histidine tag was done by biotinylated thrombin. The antigen was passed again through the nickel column and the purified tag-less antigen was gently eluted using $10 \mathrm{mM}$ imidazole. Antibodies in mice were generated as previously [65]. CD1 mice (Charles River) were inoculated intraperitoneally with $100 \mu \mathrm{g}$ of the TgTRPPL-2 peptide mixed with complete Freund's adjuvant, followed by two boosts with $50 \mu \mathrm{g}$ of the TgTRPPL-2 peptide in incomplete Freund's adjuvant. The final serum was collected by cardiac puncture after $\mathrm{CO}_{2}$ euthanasia. We created a $\alpha$ SERCA antibody for colocalization studies of the TgTRPPL-2. The phosphorylation (P) and nucleotide binding (N) domains of TgSERCA were cloned into XmaI and HindIII sites of pQE-80L plasmid for expression in Escherichia coli BL21-CodonPlus competent cells. Purified antigen was used to immunize Guinea pigs with $0.2 \mathrm{mg}$ of antigen mixed with Freund's Complete Adjuvant, followed by two boosts of $0.1 \mathrm{mg}$ antigen mixed with Freund's Incomplete Adjuvant (Sigma F5506). The resulting antibodies were used at 1:1,000 for western blots. The animal protocol used was approved by the UGA Institutional Animal Care and Use Committee (IACUC).

Western blot analysis. SDS-polyacrylamide gel electrophoresis (SDS-PAGE) followed established protocols [66]. Lysates were prepared by resuspending a pellet of $1 \times 10^{8}$ tachyzoites in $50 \mu \mathrm{L}$ of Cell Lytic ${ }^{\mathrm{M}}$ lysis buffer containing $12.5 \mathrm{U}$ benzonase and $1 \mathrm{X}$ protease cocktail inhibitor 
(P8340 Sigma). The reaction was stopped with one volume of 2\% SDS and $1 \mathrm{mM}$ EDTA. Total lysates were boiled in Laemmli sample buffer (BioRad). Immunoblotting followed established protocols using mouse anti-HA monoclonal antibody $(1: 1,000)$ (Roche). Detection was done using the Odyssey Clx LICOR system using goat anti-mouse IRDye800WC (1:10,000). Loading control for westerns were done with primary mouse-anti-tubulin antibodies at a 1:15,000 dilution and goat anti-mouse IRDDye800WC as secondary (1:10,000).

Immunofluorescence microscopy Extracellular parasites were collected and purified as previously [67]. Parasites were washed once with buffer A with glucose (BAG, $116 \mathrm{mM} \mathrm{NaCl}, 5.4$ $\mathrm{mM} \mathrm{KCl}, 0.8 \mathrm{mM} \mathrm{MgSO}_{4}, 5.5 \mathrm{mM}$ glucose and $50 \mathrm{mM}$ HEPES, $\mathrm{pH}$ 7.4) and an aliquot of $2 \times 10^{4}$ parasites was overlaid on a coverslip previously treated with poly-L-Lysine. Intracellular tachyzoites were grown on hTERT cells on coverslips. Both extracellular and intracellular parasites were fixed with $3 \%$ paraformaldehyde for $20 \mathrm{~min}$ at room temperature (RT), permeabilized with $0.3 \%$ Triton X-100, blocked with $3 \%$ bovine serum albumin (BSA), and exposed to primary antibodies (Rat $\alpha$-HA 1:100). The secondary antibodies used were goat- $\alpha$ rat Alexa Fluor 488 (Life Technologies) at a 1:1,000 dilution. For co-localization studies we used $\alpha$ Sag1 $(1: 1,000)$ as membrane marker and $\alpha$-TgSERCA as ER marker $(1: 1,000)$. Slides were examined using an Olympus IX-71 inverted fluorescence microscope with a photometric CoolSNAP HQ charge-coupled device (CCD) camera driven by DeltaVision software (Applied Precision, Seattle, WA).

Immunoprecipitation assays. Freshly lysed tachyzoites expressing TgTRPPL-2-smHA were collected and filtered through an $8 \mu \mathrm{M}$ membrane (Whatman). Tachyzoites were washed twice in BAG and resuspended in lysis buffer (50mM Tris- $\mathrm{HCl}, \mathrm{pH} 7.4,150 \mathrm{mM} \mathrm{KCl}, 1 \mathrm{mM}$ EDTA, 0.4\% NP-40) to a final concentration of $2 \times 10^{9}$ total cells. Lysis was allowed to proceed for thirty minutes at $4^{\circ} \mathrm{C}$ and cells were centrifuged at $15,000 \mathrm{x}$ g for $20 \mathrm{~min}$. Immunoprecipitation of TgTRPPL-2-smHA protein was performed using the Pierce HA Tag/Co-IP Kit (Thermo Fisher Scientific, Waltham, MA) according to manufacturer's instructions. Briefly, HA magnetic beads were washed twice in lysis buffer and mixed with the parasite lysate by vortexing for $1 \mathrm{~h}$ at RT. Beads were collected and the flow-through fraction was saved for further analysis. Beads were washed twice in wash buffer (50mM Tris-HCl, pH 7.4, $150 \mathrm{mM} \mathrm{KCl,} 1 \mathrm{mM}$ EDTA, 0.1\% NP-40) and once in $\mathrm{ddH}_{2} \mathrm{O}$ by gentle mixing. The tagged protein was recovered by mixing the beads with 1x Laemmli buffer and heated at $65^{\circ} \mathrm{C}$ for $10 \mathrm{~min}$. The supernatant was collected and used for PAGE and western blots. The corresponding band was cut and resuspended in water and analyzed using LC-Mass Spectrometry. Samples were sent to the Proteomics and Mass Spectrometry Core Facility at the University of Georgia for analysis. The average counts that were obtained from two biological samples are shown in Table S2. Proteins with counts higher than 3 are shown.

Growth and Invasion Assays. Plaque assays were done as previously described, with slight modifications [67]. Briefly, 200 egressed tachyzoites were allowed to infect confluent hTERT cells 
for 7 days. After seven days cells were fixed with ethanol and stained with crystal violet. Plaque sizes were analyzed using FIJI [68]. Invasion assay were performed as previously described, with slight modifications [28]. A subconfluent monolayer of HFF cells were infected with $2 \times 10^{7}$ tachyzoites in the presence of $1.8 \mathrm{mM}$ or $0.5 \mathrm{mM} \mathrm{Ca}^{2+}$ and placed for $20 \mathrm{~min}$ on ice and subsequently transferred for $5 \mathrm{~min}$ to a $37^{\circ} \mathrm{C}$ water bath for parasite invasion. Cells were immediately fixed with $3 \%$ paraformaldehyde for $20 \mathrm{~min}$. Extracellular parasites (attached) were stained using RabbitaSag1 (1:1,000) prior to permeabilization while intracellular parasites (invaded) were stained with Mouse $\alpha$ Sag1 (1:200). Secondary antibodies were goat- $\alpha$ rabbit Alexa Fluor $546(1: 1,000)$ and goat- $\alpha$ mouse Alexa Fluor $488(1: 1,000)$. Images were taken with an Olympus IX-71 inverted fluorescence microscope with a Photometric Cool SNAP HQ CCD camera driven by DeltaVision software (Applied Precision, Seattle, WA). Quantification was made of ten-fields of view at a 1000 magnification from three independent biological replicates. Percentage of invaded vs attached was quantified by dividing the number of parasites invaded or attached by the total parasites quantified in the field of view.

Egress experiments. hTERT cells were infected with $5 \times 10^{5}$ of RH or $\triangle$ TgTRPPL-2 tachyzoites. $24 \mathrm{~h}$ after infection parasitophorous vacuoles were observed by microscopy and washed with Ringer's buffer (155 mM NaCl, 3 mM KCl, 1 mM MgCl2, 3 mM NaH${ }_{2} \mathrm{PO}_{4} \mathrm{H}_{2} \mathrm{O}, 10 \mathrm{mM}$ HEPES, $\mathrm{pH}$ 7.3, and $5 \mathrm{mM}$ glucose). Ringer's buffer was used as extracellular buffer in the presence or absence of $1.8 \mathrm{mM} \mathrm{Ca}^{2+}$. Drugs were added in Ringer's buffer $30 \mathrm{sec}$ after imaging at the following concentrations: saponin $(0.02 \%)$ or Zaprinast $(100 \mu \mathrm{M})$. Images were acquired in a time-lapse mode with an acquisition rate of $3 \mathrm{sec}$ for 12-20 min For statistical analysis, egress time was quantified as the first parasite to egress out of the parasitophorous vacuole. Statistical analysis was done for 3 independent biological replicates and at least 5 PVs per experiment.

Cytosolic Ca $^{2+}$ measurements. Parasites were loaded with Fura2-AM as described in [36]. Briefly, fresh lysed parasites were washed twice at $1,800 \mathrm{rpm}$ for $10 \mathrm{~min}$ at room temperature in buffer A (BAG) (116 mM NaCl, $5.4 \mathrm{mM} \mathrm{KCl,} 0.8 \mathrm{mM} \mathrm{MgSO} 4,5.5 \mathrm{mM}$ d-glucose and $50 \mathrm{mM}$ Hepes, $\mathrm{pH}$ 7.4). Parasites were resuspended to a final density of $1 \times 10^{9}$ parasites $/ \mathrm{mL}$ in loading buffer (Ringer's plus $1.5 \%$ sucrose, and $5 \mu \mathrm{M}$ Fura2-AM). The suspension was incubated for 26 min at $26^{\circ} \mathrm{C}$ with mild agitation. Subsequently, the parasites were washed twice with Ringer's buffer to remove extracellular dye. Parasites were resuspended to a final density of $1 \times 10^{9}$ parasites $/ \mathrm{mL}$ in Ringer's buffer and kept in ice. For fluorescence measurements, $2 \times 10^{7}$ parasites $/ \mathrm{mL}$ were placed in a cuvette with $2.5 \mathrm{~mL}$ of Ringer's buffer. The cuvette was placed in a thermostatically controlled Hitachi F-7000 fluorescence spectrophotometer. Excitation was at 340 and $380 \mathrm{~nm}$, and emission at $510 \mathrm{~nm}$. The Fura2-AM fluorescence relationship to intracellular $\mathrm{Ca}^{2+}$ concentration $\left(\left[\mathrm{Ca}^{2+}\right]_{\mathrm{i}}\right)$ was calibrated from the ratio of $340 / 380 \mathrm{~nm}$ fluorescence values after subtraction of the background fluorescence of the cells at 340 and $380 \mathrm{~nm}$ as previously described [69]. Changes in $\left[\mathrm{Ca}^{2+}\right]_{\mathrm{i}}\left(\Delta \mathrm{F}\left[\mathrm{Ca}^{2+}\right]\right)$ were measured by subtracting the highest peak of $\mathrm{Ca}^{2+}$ in the first $20 \mathrm{~s}$ after addition of $\mathrm{Ca}^{2+}$ or $100 \mathrm{~s}$ after the addition of drugs minus the baseline. 


\section{Cell transfections and culture of $\mathrm{HEK}-3 \mathrm{KO}$ Cells.}

Total RNA of wild type $T$. gondii were extracted and reversed transcribed into cDNA. TgTRPPL2 whole cDNA was amplified using primers shown in Table S3 (Primers C1-C6). The amplified cDNA was cloned into the Zero Blunt TOPO vector using the cloning kit per manufacturers instruction. Correct insertion was verified by colony PCR using M13F and M13R primers. Restriction digests was performed to remove the insert from the vector using the following restriction enzymes: BamHI and AvrII. The purified TgTRPPL-2 $c D N A$ was ligated to linearized pCDNA 3.1 plasmid. Ligation to the vector was confirmed by PCR and sequencing. Purified TRPPL-2-pCDNA was used to co-transfect DT40-3KO cells.

HEK cells which have the 3 endogenous isoforms of the $\mathrm{IP}_{3}$ receptor knocked out were a gift from Dr. David Yule [31, 70]. The cells were maintained in Dulbecco's modified minimal essential media (DMEM) with $10 \%$ fetal bovine serum $2.5 \mu \mathrm{g} / \mathrm{ml}$ amphotericin B and $100 \mu \mathrm{g} / \mathrm{ml}$ streptomycin. Cells were transiently transfected as previously described [71] with $2.5 \mu \mathrm{g}$ of TgTRPPL-2, PC2 or RFP DNA targeted to the ER. Each plasmid DNA were diluted in $200 \mu \mathrm{L}$ of Opti-MEM with $25 \mu \mathrm{L}$ of polyethylenimine and incubated for $10 \mathrm{~min}$. The mix was then added to semi confluent HEK-3KO cells in a dropwise manner, and $24 \mathrm{~h}$ later the media was changed.

Preparation of nuclear extracts. 48 hours after transfection, cells were collected and the nucleus extracted as previously described [72]. $2 \times 10^{7}$ of transiently transfected cells were collected in ice cold PBS. Cells were spun down and washed twice in PBS and resuspended in Nuclei Isolation Solution (150 mM KCl, $250 \mathrm{mM}$ Sucrose, $10 \mathrm{mM}$ Tris-HCl, $1.4 \mathrm{mM} \beta$-mercaptoethanol, $0.2 \mathrm{mM}$ PMSF, pH 7.3). Cells were homogenized with a homogenizer and stored on ice. $100 \mu \mathrm{L}$ of nuclei were transferred to cover slips previously coated with poly-L-lysine and incubated for 20 minutes before filling the chamber with bath solution.

Patch clamp of nuclear membranes. Nuclear extract expressing TgTRPPL-2 or the control gCaMPER [73] were used for analysis. Electrical currents were recorded using Standard Wall Borosilicate Capillaries (Harvard Bioscience, Massachusetts) with 10-15 M $\Omega$ resistance. Holding potentials were maintained at $0 \mathrm{mV}$. The internal solution contained: $140 \mathrm{mM} \mathrm{KCl}$ or $\mathrm{CsCl}, 10$ $\mathrm{mM}$ HEPES, $1.8 \mathrm{mM}$ or $10 \mathrm{mM}$ free $\mathrm{Ca}^{2+}$ adjusted with EGTA. Standard Bath Solution contained the same reagents and concentrations as the pipette solution using $100 \mathrm{nM}$ of free $\mathrm{Ca}^{2+}$. The singlechannel conductance was obtained from the current-voltage relationship for each condition tested. $\mathrm{Ca}^{2+}$ currents were elicited by applying pulses from $-80 \mathrm{mV}$ up to $20 \mathrm{mV}$ for 25 seconds. Analysis of amplitude, open probability and channel conductance were done using a $45 \mathrm{kHz}$ filter. Data for recording was collected using the HEKA Electronic Patch Clamp EPC10 (Harvard Bioscience, Massachusetts).

Statistics. Statistical analyses were performed by Student's $t$-test using GraphPad PRISM version 8.2. All experimental data were analyzed from at least three independent biological replicates. 
Error bars shown represent standard error of the mean (SEM) of the biological replicates analyzed. For the electrophysiological analysis a total of 3 cells per biological replicate ( 9 total cells) were analyzed. Each cell was depolarized a total of 5 times per experimental conditions.

Acknowledgements: We thank Drs. David Yule for providing the HEK-3KO cells for electrophysiological analyses, John Boothroyd for antibodies against SAG1 and Boris Striepen for the Cosmid for complementation. Catherine Li prepared the antigen used to generate the antiSERCA antibody. The super resolution microscope is part of the Biomedical Microscopy Core (BMC) of the University of Georgia. We thank the University of Georgia Graduate School for a Summer Research Travel Grant to KMMN. This work was supported by the U.S. National Institutes of Health grant AI128356 to SNJM and R00 DK101585 to IYK. KMMN was partially supported through a fellowship funded by a T32 training grant, 5T32AI060546.

\section{Author Contributions}

KMMN, performed and coordinated most of the experiments, analyzed the data, wrote the manuscript; NC, developed the specific antibody and contributed to its validation; MAHT, developed the knockout strain and its validation; IK, writing, review, editing, analysis and interpretation of data; SNJM, coordinated the project and experiments, contributed resources and wrote the manuscript.

\section{REFERENCES}

1. Berridge MJ, Bootman MD, Roderick HL. Calcium signalling: dynamics, homeostasis and remodelling. Nat Rev Mol Cell Biol. 2003;4(7):517-29. Epub 2003/07/03. doi:

10.1038/nrm1155. PubMed PMID: 12838335.

2. Clapham DE. Calcium signaling. Cell. 2007;131(6):1047-58. doi: 10.1016/j.cell.2007.11.028. PubMed PMID: 18083096.

3. Blader IJ, Coleman BI, Chen CT, Gubbels MJ. Lytic Cycle of Toxoplasma gondii: 15 Years Later. Annu Rev Microbiol. 2015;69:463-85. Epub 2015/09/04. doi: 10.1146/annurev-micro091014-104100. PubMed PMID: 26332089; PubMed Central PMCID: PMCPMC4659696.

4. Weiss LM, Dubey JP. Toxoplasmosis: A history of clinical observations. Int J Parasitol. 2009;39(8):895-901. Epub 2009/02/17. doi: 10.1016/j.ijpara.2009.02.004. PubMed PMID: 19217908; PubMed Central PMCID: PMCPMC2704023.

5. Black MW, Boothroyd JC. Lytic cycle of Toxoplasma gondii. Microbiol Mol Biol Rev. 2000;64(3):607-23. PubMed PMID: 10974128.

6. Lourido S, Moreno SN. The calcium signaling toolkit of the Apicomplexan parasites Toxoplasma gondii and Plasmodium spp. Cell Calcium. 2015;57(3):186-93. Epub 2015/01/22. doi: 10.1016/j.ceca.2014.12.010. PubMed PMID: 25605521; PubMed Central PMCID: PMCPMC4428288.

7. Hortua Triana MA, Marquez-Nogueras KM, Vella SA, Moreno SNJ. Calcium signaling and the lytic cycle of the Apicomplexan parasite Toxoplasma gondii. Biochim Biophys Acta Mol 
Cell Res. 2018;1865(11 Pt B):1846-56. Epub 2019/04/18. doi: 10.1016/j.bbamcr.2018.08.004. PubMed PMID: 30992126; PubMed Central PMCID: PMCPMC6477927.

8. Pace DA, McKnight CA, Liu J, Jimenez V, Moreno SN. Calcium entry in Toxoplasma gondii and its enhancing effect of invasion-linked traits. J Biol Chem. 2014;289(28):1963747. Epub 2014/05/29. doi: 10.1074/jbc.M114.565390. PubMed PMID: 24867952; PubMed Central PMCID: PMCPMC4094074.

9. Vella SA, Moore, C. A., Li, Z., Hortua Triana, M.A., Potapenko, E., Moreno, S.N.J. The Role of Potassium and Host Calcium Signaling in Toxoplasma gondii egress. bioRxiv. 2020. doi: https://doi.org/10.1101/2020.03.06.980508.

10. Nilius B, Owsianik G. The transient receptor potential family of ion channels. Genome Biol. 2011;12(3):218. Epub 2011/03/16. doi: 10.1186/gb-2011-12-3-218. PubMed PMID: 21401968 ; PubMed Central PMCID: PMCPMC3129667.

11. Zhou J. Polycystins and primary cilia: primers for cell cycle progression. Annu Rev Physiol. 2009;71:83-113. Epub 2009/07/04. doi: 10.1146/annurev.physiol.70.113006.100621. PubMed PMID: 19572811.

12. Venkatachalam K, Montell C. TRP channels. Annu Rev Biochem. 2007;76:387-417. Epub 2007/06/21. doi: 10.1146/annurev.biochem.75.103004.142819. PubMed PMID: 17579562; PubMed Central PMCID: PMCPMC4196875.

13. Koulen P, Cai Y, Geng L, Maeda Y, Nishimura S, Witzgall R, et al. Polycystin-2 is an intracellular calcium release channel. Nat Cell Biol. 2002;4(3):191-7. Epub 2002/02/21. doi: 10.1038/ncb754. PubMed PMID: 11854751.

14. Samanta A, Hughes TET, Moiseenkova-Bell VY. Transient Receptor Potential (TRP) Channels. Subcell Biochem. 2018;87:141-65. Epub 2018/02/22. doi: 10.1007/978-981-107757-9_6. PubMed PMID: 29464560; PubMed Central PMCID: PMCPMC6038138.

15. Wang Z, Ng C, Liu X, Wang Y, Li B, Kashyap P, et al. The ion channel function of polycystin-1 in the polycystin-1/polycystin-2 complex. EMBO Rep. 2019;20(11):e48336. Epub 2019/08/24. doi: 10.15252/embr.201948336. PubMed PMID: 31441214; PubMed Central PMCID: PMCPMC6832002.

16. Wolstenholme AJ, Williamson SM, Reaves BJ. TRP channels in parasites. Adv Exp Med Biol. 2011;704:359-71. Epub 2011/02/04. doi: 10.1007/978-94-007-0265-3_20. PubMed PMID: 21290306.

17. Prole DL, Taylor CW. Identification of intracellular and plasma membrane calcium channel homologues in pathogenic parasites. PloS one. 2011;6(10):e26218. Epub 2011/10/25. doi: 10.1371/journal.pone.0026218. PubMed PMID: 22022573; PubMed Central PMCID: PMC3194816.

18. Boucher C, Sandford R. Autosomal dominant polycystic kidney disease (ADPKD, MIM 173900, PKD1 and PKD2 genes, protein products known as polycystin-1 and polycystin-2). Eur J Hum Genet. 2004;12(5):347-54. Epub 2004/02/12. doi: 10.1038/sj.ejhg.5201162. PubMed PMID: 14872199.

19. Hortua Triana MA, Marquez-Nogueras KM, Chang L, Stasic AJ, Li C, Spiegel KA, et al. Tagging of Weakly Expressed Toxoplasma gondii Calcium-Related Genes with HighAffinity Tags. J Eukaryot Microbiol. 2018;65(5):709-21. Epub 2018/04/20. doi: 10.1111/jeu.12626. PubMed PMID: 29672999; PubMed Central PMCID: PMCPMC6175649.

20. Montell C. The TRP superfamily of cation channels. Sci STKE. 2005;2005(272):re3. Epub 2005/02/25. doi: 10.1126/stke.2722005re3. PubMed PMID: 15728426. 
21. Omasits U, Ahrens CH, Muller S, Wollscheid B. Protter: interactive protein feature visualization and integration with experimental proteomic data. Bioinformatics. 2014;30(6):884-6. Epub 2013/10/29. doi: 10.1093/bioinformatics/btt607. PubMed PMID: 24162465.

22. Soding J. Protein homology detection by HMM-HMM comparison. Bioinformatics. 2005;21(7):951-60. Epub 2004/11/09. doi: 10.1093/bioinformatics/bti125. PubMed PMID: 15531603.

23. Sheiner L, Demerly JL, Poulsen N, Beatty WL, Lucas O, Behnke MS, et al. A systematic screen to discover and analyze apicoplast proteins identifies a conserved and essential protein import factor. PLoS Pathog. 2011;7(12):e1002392. Epub 2011/12/07. doi: 10.1371/journal.ppat.1002392. PubMed PMID: 22144892; PubMed Central PMCID: PMCPMC3228799.

24. Shen PS, Yang X, DeCaen PG, Liu X, Bulkley D, Clapham DE, et al. The Structure of the Polycystic Kidney Disease Channel PKD2 in Lipid Nanodiscs. Cell. 2016;167(3):763-73 e11. Epub 2016/10/22. doi: 10.1016/j.cell.2016.09.048. PubMed PMID: 27768895; PubMed Central PMCID: PMCPMC6055481.

25. Su Q, Hu F, Liu Y, Ge X, Mei C, Yu S, et al. Cryo-EM structure of the polycystic kidney disease-like channel PKD2L1. Nat Commun. 2018;9(1):1192. Epub 2018/03/24. doi: 10.1038/s41467-018-03606-0. PubMed PMID: 29567962; PubMed Central PMCID: PMCPMC5864754.

26. Merrick D, Bertuccio CA, Chapin HC, Lal M, Chauvet V, Caplan MJ. Polycystin-1 cleavage and the regulation of transcriptional pathways. Pediatr Nephrol. 2014;29(4):505-11. Epub 2013/07/05. doi: 10.1007/s00467-013-2548-y. PubMed PMID: 23824180; PubMed Central PMCID: PMCPMC3844055.

27. Vinayak S, Brooks CF, Naumov A, Suvorova ES, White MW, Striepen B. Genetic manipulation of the Toxoplasma gondii genome by fosmid recombineering. mBio. 2014;5(6):e02021. Epub 2014/12/04. doi: 10.1128/mBio.02021-14. PubMed PMID: 25467441 ; PubMed Central PMCID: PMCPMC4324243.

28. Kafsack BF, Beckers C, Carruthers VB. Synchronous invasion of host cells by Toxoplasma gondii. Mol Biochem Parasitol. 2004;136(2):309-11. Epub 2004/10/14. doi: 10.1016/j.molbiopara.2004.04.004. PubMed PMID: 15478810.

29. Petri ET, Celic A, Kennedy SD, Ehrlich BE, Boggon TJ, Hodsdon ME. Structure of the EFhand domain of polycystin-2 suggests a mechanism for Ca2+-dependent regulation of polycystin-2 channel activity. Proc Natl Acad Sci U S A. 2010;107(20):9176-81. Epub 2010/05/05. doi: 10.1073/pnas.0912295107. PubMed PMID: 20439752; PubMed Central PMCID: PMCPMC2889120.

30. Sidik SM, Hortua Triana MA, Paul AS, El Bakkouri M, Hackett CG, Tran F, et al. Using a Genetically Encoded Sensor to Identify Inhibitors of Toxoplasma gondii Ca2+ Signaling. J Biol Chem. 2016;291(18):9566-80. Epub 2016/03/05. doi: 10.1074/jbc.M115.703546. PubMed PMID: 26933036; PubMed Central PMCID: PMCPMC4850295.

31. Alzayady KJ, Wang L, Chandrasekhar R, Wagner LE, 2nd, Van Petegem F, Yule DI. Defining the stoichiometry of inositol 1,4,5-trisphosphate binding required to initiate $\mathrm{Ca} 2+$ release. Sci Signal. 2016;9(422):ra35. Epub 2016/04/07. doi: 10.1126/scisignal.aad6281. PubMed PMID: 27048566; PubMed Central PMCID: PMCPMC4850551.

32. Celic A, Petri ET, Demeler B, Ehrlich BE, Boggon TJ. Domain mapping of the polycystin-2 C-terminal tail using de novo molecular modeling and biophysical analysis. J Biol Chem. 
2008;283(42):28305-12. Epub 2008/08/13. doi: 10.1074/jbc.M802743200. PubMed PMID: 18694932; PubMed Central PMCID: PMCPMC2568934.

33. Almog M, Korngreen A. Characterization of voltage-gated $\mathrm{Ca}(2+)$ conductances in layer 5 neocortical pyramidal neurons from rats. PLoS One. 2009;4(4):e4841. Epub 2009/04/02. doi: 10.1371/journal.pone.0004841. PubMed PMID: 19337371; PubMed Central PMCID: PMCPMC2659773.

34. Wu LG, Westenbroek RE, Borst JG, Catterall WA, Sakmann B. Calcium channel types with distinct presynaptic localization couple differentially to transmitter release in single calyxtype synapses. J Neurosci. 1999;19(2):726-36. Epub 1999/01/09. PubMed PMID: 9880593; PubMed Central PMCID: PMCPMC6782194.

35. Harteneck C, Frenzel H, Kraft R. N-(p-amylcinnamoyl)anthranilic acid (ACA): a phospholipase A(2) inhibitor and TRP channel blocker. Cardiovasc Drug Rev. 2007;25(1):61-75. Epub 2007/04/21. doi: 10.1111/j.1527-3466.2007.00005.x. PubMed PMID: 17445088.

36. Moreno SN, Zhong L. Acidocalcisomes in Toxoplasma gondii tachyzoites. Biochem J. 1996;313 ( Pt 2):655-9. PubMed PMID: 8573106.

37. Lovett JL, Marchesini N, Moreno SN, Sibley LD. Toxoplasma gondii microneme secretion involves intracellular $\mathrm{Ca}(2+)$ release from inositol 1,4,5-triphosphate (IP(3))/ryanodinesensitive stores. J Biol Chem. 2002;277(29):25870-6. PubMed PMID: 12011085.

38. Lovett JL, Sibley LD. Intracellular calcium stores in Toxoplasma gondii govern invasion of host cells. J Cell Sci. 2003;116(Pt 14):3009-16. PubMed PMID: 12783987.

39. Bezzerides VJ, Ramsey IS, Kotecha S, Greka A, Clapham DE. Rapid vesicular translocation and insertion of TRP channels. Nat Cell Biol. 2004;6(8):709-20. Epub 2004/07/20. doi: 10.1038/ncb1150. PubMed PMID: 15258588.

40. Cai Y, Maeda Y, Cedzich A, Torres VE, Wu G, Hayashi T, et al. Identification and characterization of polycystin-2, the PKD2 gene product. J Biol Chem. 1999;274(40):2855765. Epub 1999/09/25. doi: 10.1074/jbc.274.40.28557. PubMed PMID: 10497221.

41. Nagamune K, Beatty WL, Sibley LD. Artemisinin induces calcium-dependent protein secretion in the protozoan parasite Toxoplasma gondii. Eukaryotic cell. 2007;6(11):2147-56. Epub 2007/09/04. doi: 10.1128/EC.00262-07. PubMed PMID: 17766463; PubMed Central PMCID: PMC2168421.

42. Sagara Y, Inesi G. Inhibition of the sarcoplasmic reticulum $\mathrm{Ca} 2+$ transport ATPase by thapsigargin at subnanomolar concentrations. J Biol Chem. 1991;266(21):13503-6. Epub 1991/07/25. PubMed PMID: 1830305.

43. Thastrup O, Cullen PJ, Drobak BK, Hanley MR, Dawson AP. Thapsigargin, a tumor promoter, discharges intracellular $\mathrm{Ca} 2+$ stores by specific inhibition of the endoplasmic reticulum Ca2(+)-ATPase. Proc Natl Acad Sci U S A. 1990;87(7):2466-70. Epub 1990/04/01. PubMed PMID: 2138778; PubMed Central PMCID: PMCPMC53710.

44. Guerrero-Hernandez A, Dagnino-Acosta A, Verkhratsky A. An intelligent sarco-endoplasmic reticulum $\mathrm{Ca} 2+$ store: release and leak channels have differential access to a concealed $\mathrm{Ca} 2+$ pool. Cell Calcium. 2010;48(2-3):143-9. Epub 2010/09/08. doi: 10.1016/j.ceca.2010.08.001. PubMed PMID: 20817294.

45. Carreras-Sureda A, Pihan P, Hetz C. Calcium signaling at the endoplasmic reticulum: finetuning stress responses. Cell Calcium. 2018;70:24-31. Epub 2017/10/22. doi: 10.1016/j.ceca.2017.08.004. PubMed PMID: 29054537. 
46. Miranda K, Pace DA, Cintron R, Rodrigues JC, Fang J, Smith A, et al. Characterization of a novel organelle in Toxoplasma gondii with similar composition and function to the plant vacuole. Molecular microbiology. 2010;76(6):1358-75. Epub 2010/04/20. doi:

10.1111/j.1365-2958.2010.07165.x. PubMed PMID: 20398214; PubMed Central PMCID: PMC2907454.

47. Ta CM, Vien TN, Ng LCT, DeCaen PG. Structure and function of polycystin channels in primary cilia. Cell Signal. 2020;72:109626. Epub 2020/04/07. doi: 10.1016/j.cellsig.2020.109626. PubMed PMID: 32251715; PubMed Central PMCID: PMCPMC7373203.

48. Kleene SJ, Kleene NK. The native TRPP2-dependent channel of murine renal primary cilia. Am J Physiol Renal Physiol. 2017;312(1):F96-F108. Epub 2016/10/21. doi:

10.1152/ajprenal.00272.2016. PubMed PMID: 27760766; PubMed Central PMCID: PMCPMC5283891.

49. Liu X, Vien T, Duan J, Sheu SH, DeCaen PG, Clapham DE. Polycystin-2 is an essential ion channel subunit in the primary cilium of the renal collecting duct epithelium. Elife. 2018;7. Epub 2018/02/15. doi: 10.7554/eLife.33183. PubMed PMID: 29443690; PubMed Central PMCID: PMCPMC5812715.

50. Vien TN, Wang J, Ng LCT, Cao E, DeCaen PG. Molecular dysregulation of ciliary polycystin-2 channels caused by variants in the TOP domain. Proc Natl Acad Sci U S A. 2020;117(19):10329-38. Epub 2020/04/26. doi: 10.1073/pnas.1920777117. PubMed PMID: 32332171; PubMed Central PMCID: PMCPMC7229662.

51. Kuo IY, Keeler C, Corbin R, Celic A, Petri ET, Hodsdon ME, et al. The number and location of EF hand motifs dictates the calcium dependence of polycystin-2 function. FASEB J. 2014;28(5):2332-46. Epub 2014/02/22. doi: 10.1096/fj.13-247106. PubMed PMID: 24558196; PubMed Central PMCID: PMCPMC3986840.

52. Yang Y, Keeler C, Kuo IY, Lolis EJ, Ehrlich BE, Hodsdon ME. Oligomerization of the polycystin-2 C-terminal tail and effects on its Ca2+-binding properties. J Biol Chem. 2015;290(16):10544-54. Epub 2015/02/27. doi: 10.1074/jbc.M115.641803. PubMed PMID: 25716316; PubMed Central PMCID: PMCPMC4400361.

53. DeCaen PG, Liu X, Abiria S, Clapham DE. Atypical calcium regulation of the PKD2-L1 polycystin ion channel. Elife. 2016;5. Epub 2016/06/28. doi: 10.7554/eLife.13413. PubMed PMID: 27348301; PubMed Central PMCID: PMCPMC4922860.

54. Chen XZ, Vassilev PM, Basora N, Peng JB, Nomura H, Segal Y, et al. Polycystin-L is a calcium-regulated cation channel permeable to calcium ions. Nature. 1999;401(6751):383-6. Epub 1999/10/12. doi: 10.1038/43907. PubMed PMID: 10517637.

55. Burgoyne T, Patel S, Eden ER. Calcium signaling at ER membrane contact sites. Biochim Biophys Acta. 2015;1853(9):2012-7. Epub 2015/02/11. doi: 10.1016/j.bbamcr.2015.01.022. PubMed PMID: 25662816.

56. Berridge MJ. Calcium microdomains: organization and function. Cell Calcium. 2006;40(56):405-12. Epub 2006/10/13. doi: 10.1016/j.ceca.2006.09.002. PubMed PMID: 17030366.

57. Mulier M, Vriens J, Voets T. TRP channel pores and local calcium signals. Cell Calcium. 2017;66:19-24. Epub 2017/08/16. doi: 10.1016/j.ceca.2017.04.007. PubMed PMID: 28807146.

58. Liu X, Ong HL, Ambudkar I. TRP Channel Involvement in Salivary Glands-Some Good, Some Bad. Cells. 2018;7(7). Epub 2018/07/13. doi: 10.3390/cells7070074. PubMed PMID: 29997338; PubMed Central PMCID: PMCPMC6070825. 
59. Farwell DG, Shera KA, Koop JI, Bonnet GA, Matthews CP, Reuther GW, et al. Genetic and epigenetic changes in human epithelial cells immortalized by telomerase. Am J Pathol. 2000;156(5):1537-47. Epub 2000/05/04. doi: 10.1016/S0002-9440(10)65025-0. PubMed PMID: 10793065 ; PubMed Central PMCID: PMCPMC1876907.

60. Meissner M, Brecht S, Bujard H, Soldati D. Modulation of myosin A expression by a newly established tetracycline repressor-based inducible system in Toxoplasma gondii. Nucleic Acids Res. 2001;29(22):E115. Epub 2001/11/20. doi: 10.1093/nar/29.22.e115. PubMed PMID: 11713335; PubMed Central PMCID: PMCPMC92585.

61. Fox BA, Ristuccia JG, Gigley JP, Bzik DJ. Efficient gene replacements in Toxoplasma gondii strains deficient for nonhomologous end joining. Eukaryot Cell. 2009;8(4):520-9. Epub 2009/02/17. doi: 10.1128/EC.00357-08. PubMed PMID: 19218423; PubMed Central PMCID: PMCPMC2669201.

62. Shen B, Brown K, Long S, Sibley LD. Development of CRISPR/Cas9 for Efficient Genome Editing in Toxoplasma gondii. Methods Mol Biol. 2017;1498:79-103. Epub 2016/10/07. doi: 10.1007/978-1-4939-6472-7_6. PubMed PMID: 27709570.

63. Livak KJ, Flood SJ, Marmaro J, Giusti W, Deetz K. Oligonucleotides with fluorescent dyes at opposite ends provide a quenched probe system useful for detecting PCR product and nucleic acid hybridization. PCR Methods Appl. 1995;4(6):357-62. Epub 1995/06/01. doi: 10.1101/gr.4.6.357. PubMed PMID: 7580930.

64. Chasen NM, Asady B, Lemgruber L, Vommaro RC, Kissinger JC, Coppens I, et al. A Glycosylphosphatidylinositol-Anchored Carbonic Anhydrase-Related Protein of Toxoplasma gondii Is Important for Rhoptry Biogenesis and Virulence. mSphere. 2017;2(3). Epub 2017/05/23. doi: 10.1128/mSphere.00027-17. PubMed PMID: 28529974; PubMed Central PMCID: PMCPMC5437132.

65. Chasen NM, Stasic AJ, Asady B, Coppens I, Moreno SNJ. The Vacuolar Zinc Transporter TgZnT Protects Toxoplasma gondii from Zinc Toxicity. mSphere. 2019;4(3). Epub 2019/05/24. doi: 10.1128/mSphere.00086-19. PubMed PMID: 31118298; PubMed Central PMCID: PMCPMC6531880.

66. Laemmli UK. Cleavage of structural proteins during the assembly of the head of bacteriophage T4. Nature. 1970;227(5259):680-5. Epub 1970/08/15. doi: 10.1038/227680a0. PubMed PMID: 5432063.

67. Liu J, Pace D, Dou Z, King TP, Guidot D, Li ZH, et al. A vacuolar-H(+) -pyrophosphatase (TgVP1) is required for microneme secretion, host cell invasion, and extracellular survival of Toxoplasma gondii. Mol Microbiol. 2014;93(4):698-712. Epub 2014/07/01. doi: 10.1111/mmi.12685. PubMed PMID: 24975633; PubMed Central PMCID: PMCPMC4159726.

68. Schindelin J, Arganda-Carreras I, Frise E, Kaynig V, Longair M, Pietzsch T, et al. Fiji: an open-source platform for biological-image analysis. Nat Methods. 2012;9(7):676-82. Epub 2012/06/30. doi: 10.1038/nmeth.2019. PubMed PMID: 22743772; PubMed Central PMCID: PMCPMC3855844.

69. Grynkiewicz G, Poenie M, Tsien RY. A new generation of Ca2+ indicators with greatly improved fluorescence properties. J Biol Chem. 1985;260(6):3440-50. PubMed PMID: 3838314.

70. Alzayady KJ, Wagner LE, 2nd, Chandrasekhar R, Monteagudo A, Godiska R, Tall GG, et al. Functional inositol 1,4,5-trisphosphate receptors assembled from concatenated homo- and heteromeric subunits. J Biol Chem. 2013;288(41):29772-84. Epub 2013/08/21. doi: 
10.1074/jbc.M113.502203. PubMed PMID: 23955339; PubMed Central PMCID: PMCPMC3795275.

71. Longo PA, Kavran JM, Kim MS, Leahy DJ. Transient mammalian cell transfection with polyethylenimine (PEI). Methods Enzymol. 2013;529:227-40. Epub 2013/09/10. doi: 10.1016/B978-0-12-418687-3.00018-5. PubMed PMID: 24011049; PubMed Central PMCID: PMCPMC4012321.

72. Mak DO, Vais H, Cheung KH, Foskett JK. Nuclear patch-clamp electrophysiology of Ca2+ channels. Cold Spring Harb Protoc. 2013;2013(9):885-91. Epub 2013/09/05. doi: 10.1101/pdb.prot073064. PubMed PMID: 24003194; PubMed Central PMCID: PMCPMC3984033.

73. Henderson MJ, Baldwin HA, Werley CA, Boccardo S, Whitaker LR, Yan X, et al. A Low Affinity GCaMP3 Variant (GCaMPer) for Imaging the Endoplasmic Reticulum Calcium Store. PLoS One. 2015;10(10):e0139273. Epub 2015/10/10. doi: 10.1371/journal.pone.0139273. PubMed PMID: 26451944; PubMed Central PMCID: PMCPMC4599735. 

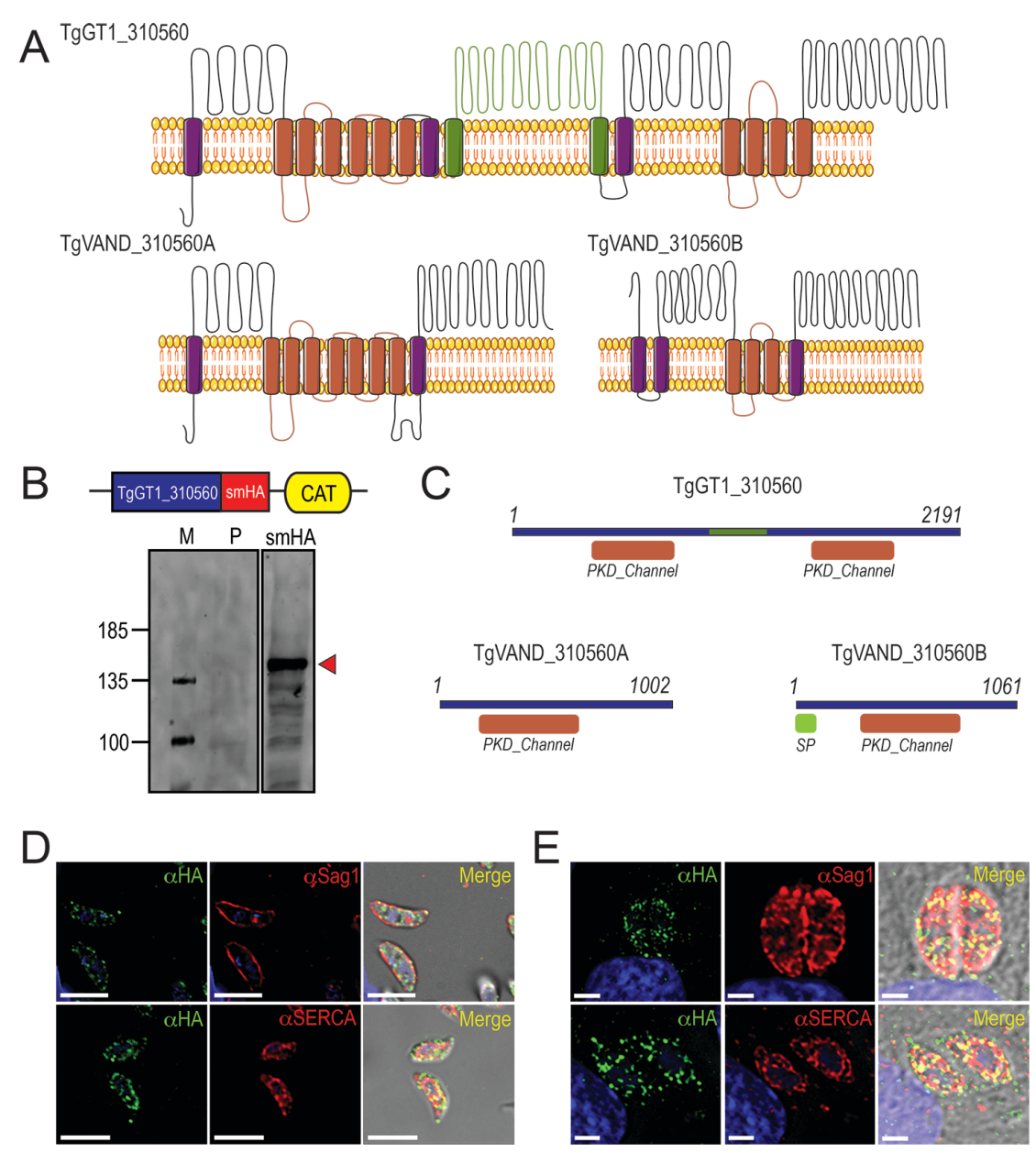

$\mathrm{F}$
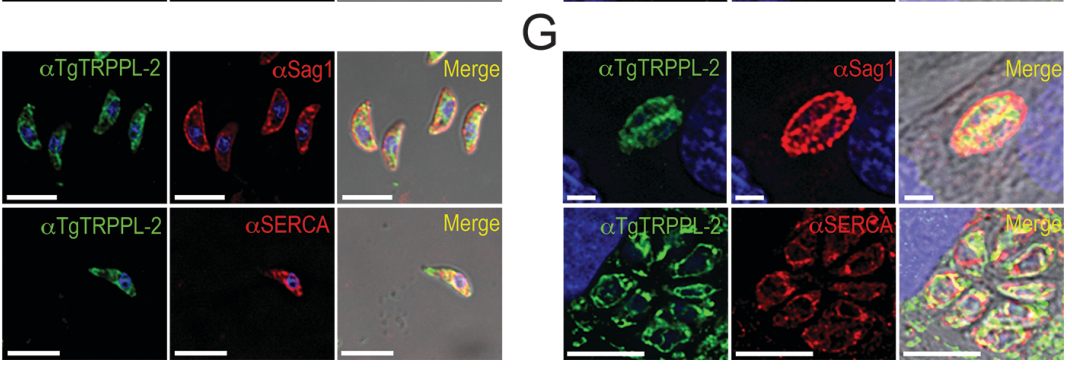

Figure 1. TgTRPPL-2 localizes to the plasma membrane (PM) and ER of T. gondii. A. Predicted topology for TgTRPPL-2 in GT1 and VAND strains. Model was generated with the Protter application [21]. The PKD Domain is highlighted in orange. The domain used to generate antibodies is highlighted in green. B. Schematic representation of C-terminal tagging of TgTRPPL-2 in Tati $\Delta$ Ku80 parasites and western blots of TgTRPPL-2-smHA membranes using $\alpha \mathrm{HA}(1: 1,000)$ showing a major band at approximately $150 \mathrm{kDa}$ (red arrowhead). C. Schematic representation of the InterPro Domain annotation of TgTRPPL-2 in GT1 and VAND strains. D. IFAs of extracellular tachyzoites using $\alpha H A$ antibody show vesicular staining close to the PM and intracellular. Co-localization with $\alpha$ SAG1 and $\alpha$ SERCA show partial co-localization with both markers. E. IFAs of intracellular tachyzoites with $\alpha \mathrm{HA}(1: 100), \alpha \operatorname{Sag} 1(1: 1,000)$ and $\alpha \operatorname{SERCA}(1: 1,000)$ showing co-localization at the PM and ER. F-G. IFAs of extracellular and intracellular tachyzoites respectively with $\alpha$ TRPPL-2 $(1: 1,000)$ showing labeling of the protein at the periphery, co-localized with $\alpha$ SAG1 $(1: 1,000)$ and ER co-localized with $\alpha \operatorname{TgSERCA}(1: 1,000)$. 
A

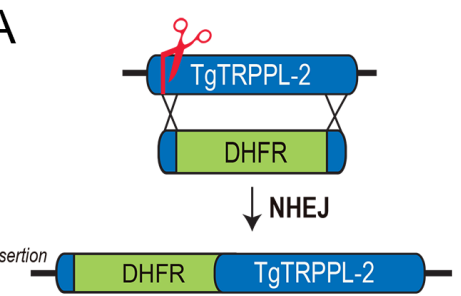

B

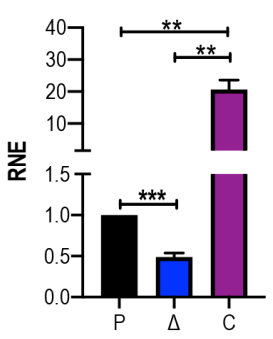

$\mathrm{D}$
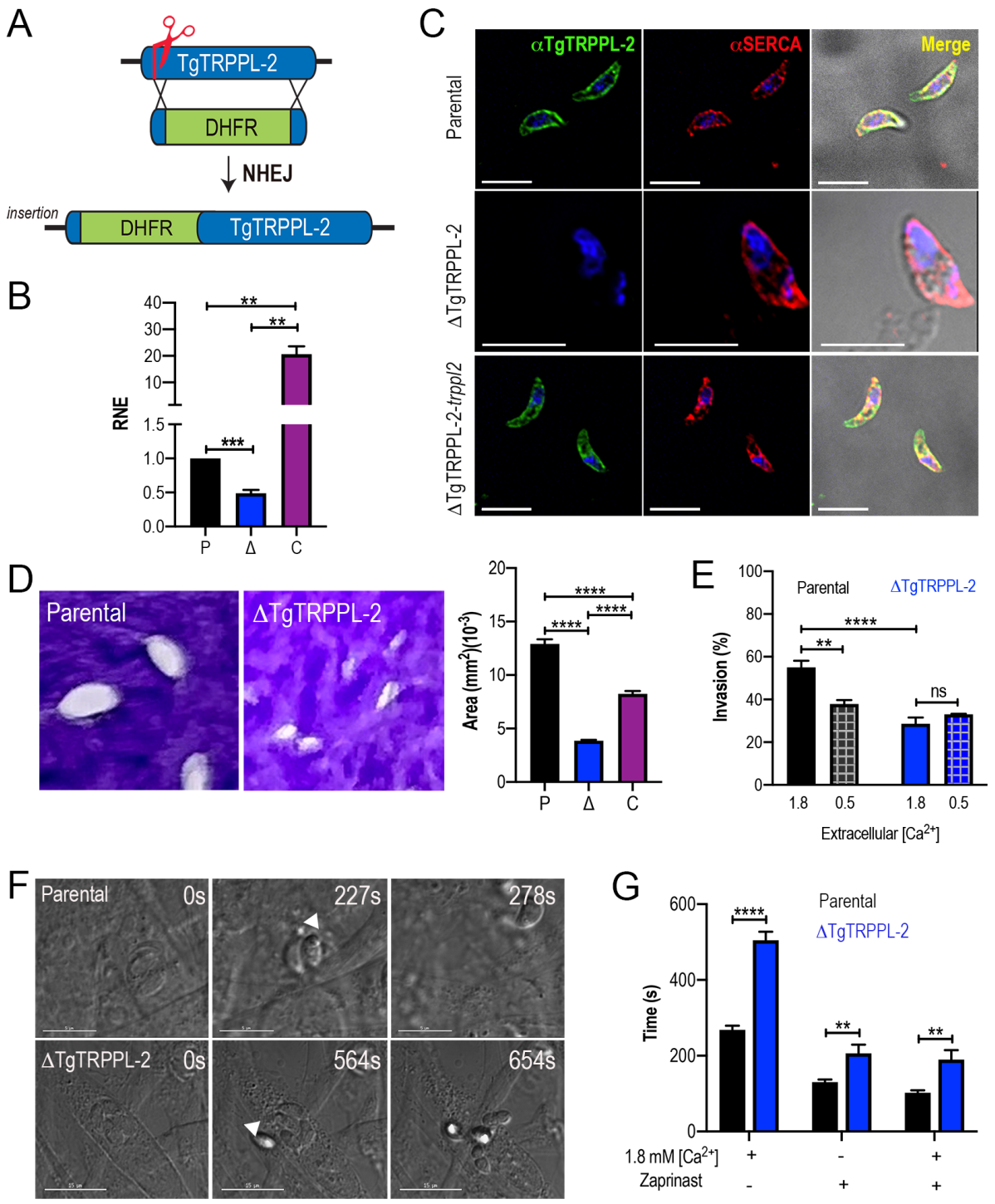

G

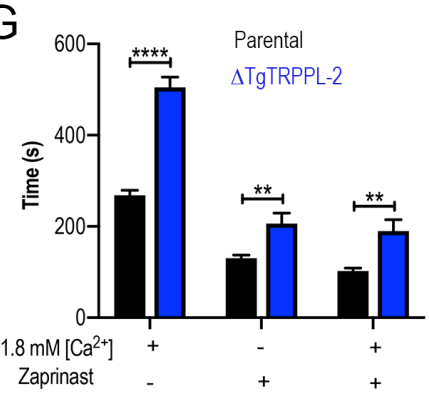

Figure 2. The role of TgTRPPL-2 in $T$. gondii growth. Schematic representation of the generation of $\triangle T g T R P P L-2$ in the T. gondii RH strain. B. qPCR of total RNA from $\triangle T g T R P P L-2, \triangle T g T R P P L-2$-trppl2 and parental strains using primers upstream and downstream of the insertion site of the DHFR cassette. C. IFAs of extracellular parasites showing PM labeling with $\alpha$ TgTRPPL2 $(1: 1,000)$ and co-localization with $\alpha$ SERCA $(1: 1,000)$. D. Plaque assays of parental $(\mathrm{P}), \triangle T g T R P P L-2(\Delta)$ and $\triangle T g T R P P L-2$-trppl2 (C) parasites. Quantification of plaque sizes from three independent biological experiments using Student's $t$-test. **** $p<$ 0.0001. E. Red green assays of parental, $\triangle T g T R P P L-2$ and $\triangle T g T R P P L-2$-trppl2 cells quantifying invaded and attached intracellular parasites. Assays were done at two concentrations of extracellular $\mathrm{Ca}^{2+}: 0.5$ and $1.8 \mathrm{mM}$. Values are means \pm SEM. $* * p<0.001, * * * * p<0.0001$. F. Time to egress stimulated by saponin $/ \mathrm{Ca}^{2+}$ at 1.8 $\mathrm{mM}$ extracellular $\mathrm{Ca}^{2+}$ of both parental or the $\triangle T g T R P P L-2$ mutant. G. Statistical analysis of average egress time stimulated by saponin or Zaprinast in the presence or absence of extracellular $\mathrm{Ca}^{2+}$. Analysis was performed from three independent biological replicates using Student's t-test. Values are means \pm SEM, $* * p$ $<0.003, * * * * p<0.0001$. Black bars represent parental strain, blue bars represent $\Delta$ TgTRPPL-2. 

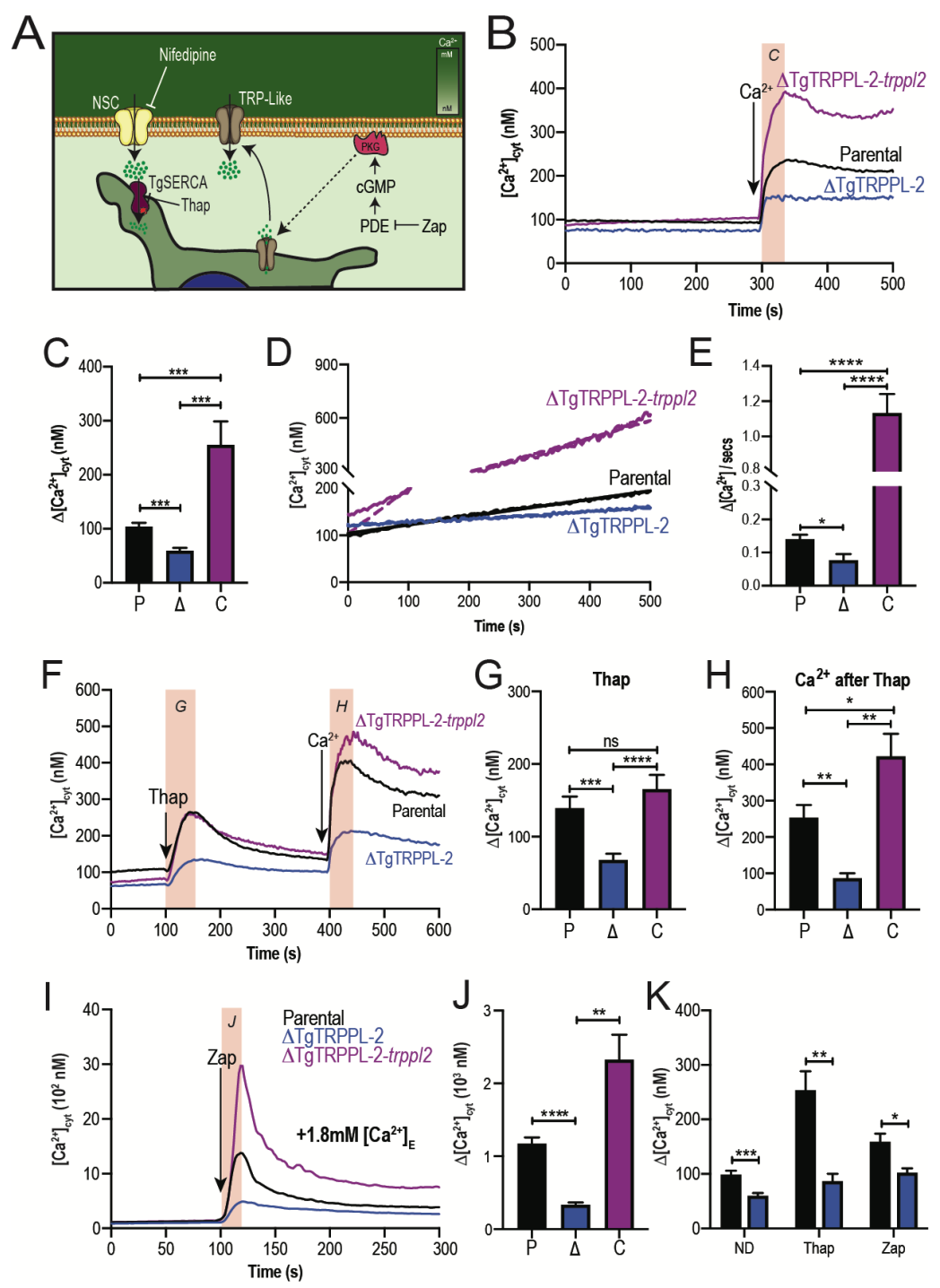

Figure 3. The role of TgTRPPL-2 in PM Ca ${ }^{2+}$ influx. A. Scheme showing the mechanism of $\mathrm{Ca}^{2+}$ influx and how cytosolic $\mathrm{Ca}^{2+}$ may activate the PM channel ( $\mathrm{Ca}^{2+}$-activated Calcium Entry). NSC, Nifedipine-Sensitive Channel; PKG, Protein Kinase G; PDE, phosphodiesterase; Thap, Thapsigargin. B. Cytosolic $\mathrm{Ca}^{2+}$ measurements of Fura2 loaded tachyzoites of the parental (P), $\triangle T g T R P P L-2(\triangle)$ and $\triangle T g T R P P L-2-t r p p l 2(\mathrm{C})$ lines. The buffer contains $100 \mu \mathrm{M}$ EGTA and at $300 \mathrm{sec}, 1.8 \mathrm{mM} \mathrm{Ca}^{2+}$ was added to the suspension. The orange box indicates the area used for the quantification presented in C.C. Quantification and statistical analysis of the change in cytosolic $\mathrm{Ca}^{2+}$ during the first $20 \mathrm{~s}$ after addition of extracellular $\mathrm{Ca}^{2+}$. $* * * p<0.0002$. D. Cytosolic $\mathrm{Ca}^{2+}$ increase of parasites preincubated with $1.8 \mathrm{mM} \mathrm{Ca}^{2+}$. E. Quantification and statistical analysis of the slope from D. **** $p<0.0001$. F. $\mathrm{Ca}^{2+}$ efflux after adding Thap $(1 \mu \mathrm{M})$ followed by $\mathrm{Ca}^{2+}$ influx after the addition of $1.8 \mathrm{mM}$ extracellular $\mathrm{Ca}^{2+}$. The orange boxes indicate the area used for the quantification presented in $G$ and $H$. G. Quantification and statistical analysis of the change in cytosolic $\mathrm{Ca}^{2+} 50 \mathrm{~s}$ after the addition of Thap (Thap) and (H) $20 \mathrm{~s}$ after the addition of 1.8 $\mathrm{mM}$ of $\mathrm{Ca}^{2+}\left(\mathrm{Ca}^{2+}\right.$ after Thap $)$. *** $p<0.0008$, **** $p<0.0001$. G. Cytosolic $\mathrm{Ca}^{2+}$ increase stimulated by Zaprinast $(100 \mu \mathrm{M})$ in the presence of $1.8 \mathrm{mM}$ extracellular $\mathrm{Ca}^{2+}$. J. Quantification and statistical analysis of cytosolic $\mathrm{Ca}^{2+}$ increase during the first $15 \mathrm{~s}$ after adding Zaprinast $(100 \mu \mathrm{M})($ Orange box, in I). $* * p<0.001$, $* * * * p<0.0001$. K. Quantification and statistical analysis of $\mathrm{Ca}^{2+}$ influx during the $20 \mathrm{~s}$ after adding $\mathrm{Ca}^{2+}$ without additions (ND) or after adding Thap or Zap. $* \mathrm{p}<0.02, * * \mathrm{p}<0.005, * * * \mathrm{p}<0.0008$. Statistical analysis for all experiments were done from at least three independent trials using student's t-test. 


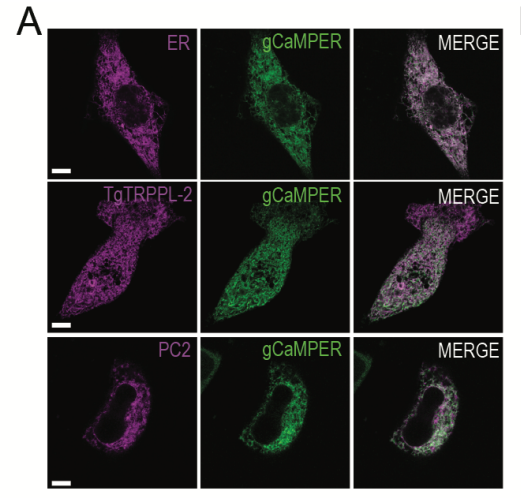

$\mathrm{B}$
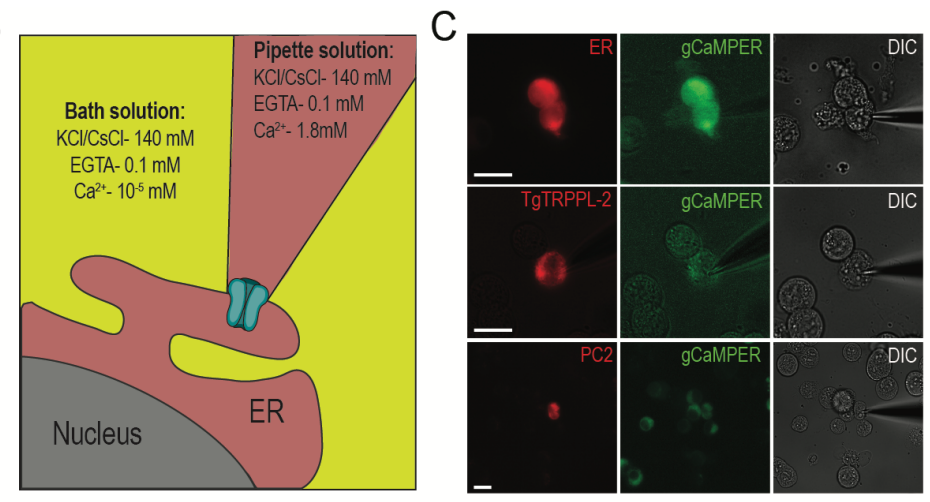

D control

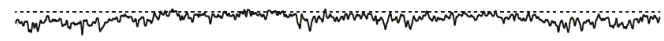
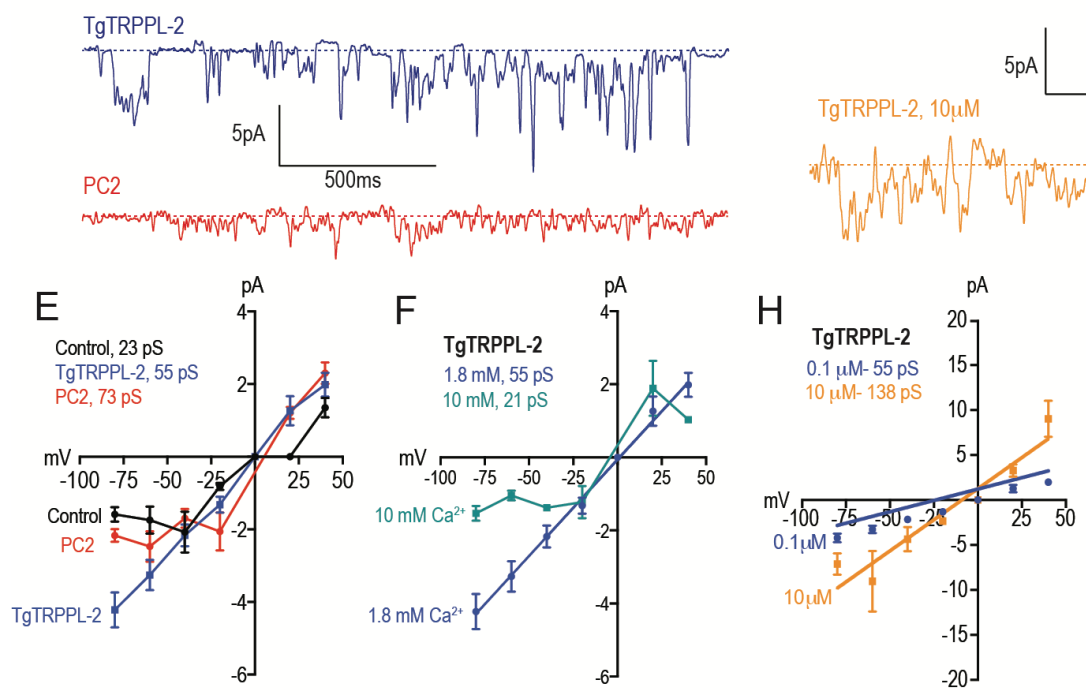

G TgTRPPL-2, 0.1uM

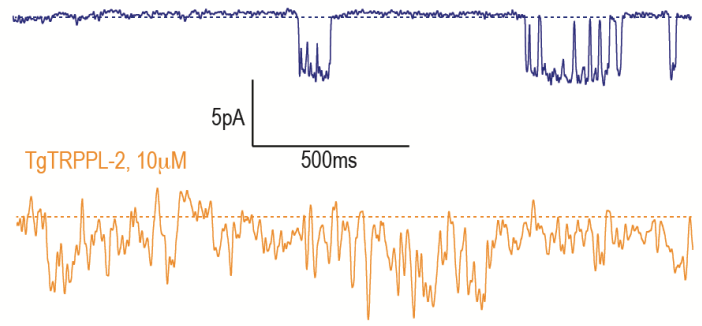

I

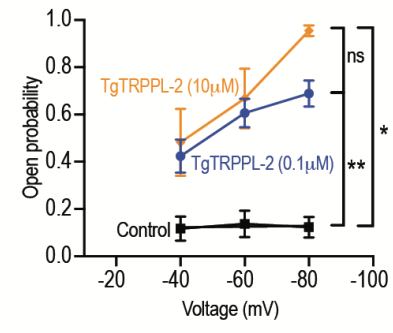

Figure 4. Characterization of TgTRPPL-2 expressed in HEK-3KO cells. A. Images of HEK-3KO cells expressing an ER-marker, PC2 or TgTRPPL-2 with the genetic calcium indicator gCAMPER. B. Schematic representation of nuclear-patch clamp. Ionic composition and concentration for bath and pipette solutions are shown. C. Patched nuclear-extract expressing ER-marker, PC2 and TgTRPPL-2 with the genetic calcium indicator gCaMPER. D. Representative tracing from control, TgTRPPL-2 or PC2 expressing cells showing the currents recorded in the presence of $1.8 \mathrm{mM}$ luminal $\mathrm{Ca}^{2+}$ in a potassium chloride solution. Tracings represent approximately $2 \mathrm{sec}$ from a $25 \mathrm{sec}$ analysis and filtered using $45 \mathrm{kHz}$. E. Current-Voltage relationship comparing single-channel conductance of control, PC2 and TgTRPPL-2 expressing cells from D. Inset, calculated channel conductance for Control, TgTRPP-L 2 and PC2. F. Current-Voltage relationship comparing single-channel conductance of TgTRPPL-2 expressing cells at 1.8 and $10 \mathrm{mM}\left[\mathrm{Ca}^{2+}\right]$ inside the pipette. Inset, calculated channel conductance for the conditions analyzed. G. Representative tracing of currents recorded from TgTRPPL-2 expressing cells using different concentration of $\left[\mathrm{Ca}^{2+}\right]$ in the bath solution (Solution A vs. Solution B) (Table S4). Tracings represent approximately $2 \mathrm{sec}$ from a $25 \mathrm{sec}$ analysis and filtered using $45 \mathrm{kHz}$. H. Current-voltage relationship comparing single-channel conductance of TgTRPPL-2 expressing cells at 0.1 and $10 \mu \mathrm{M}\left[\mathrm{Ca}^{2+}\right]$ in the bath solution. Inset, calculated channel conductance for the different $\left[\mathrm{Ca}^{2+}\right]$. I. Open probability of control and TgTRPPL-2 expressing cells in the presence of different $\left[\mathrm{Ca}^{2+}\right]$ in the bath solution in comparison to the Control. * $p<0.01, * * p<0.001$. 

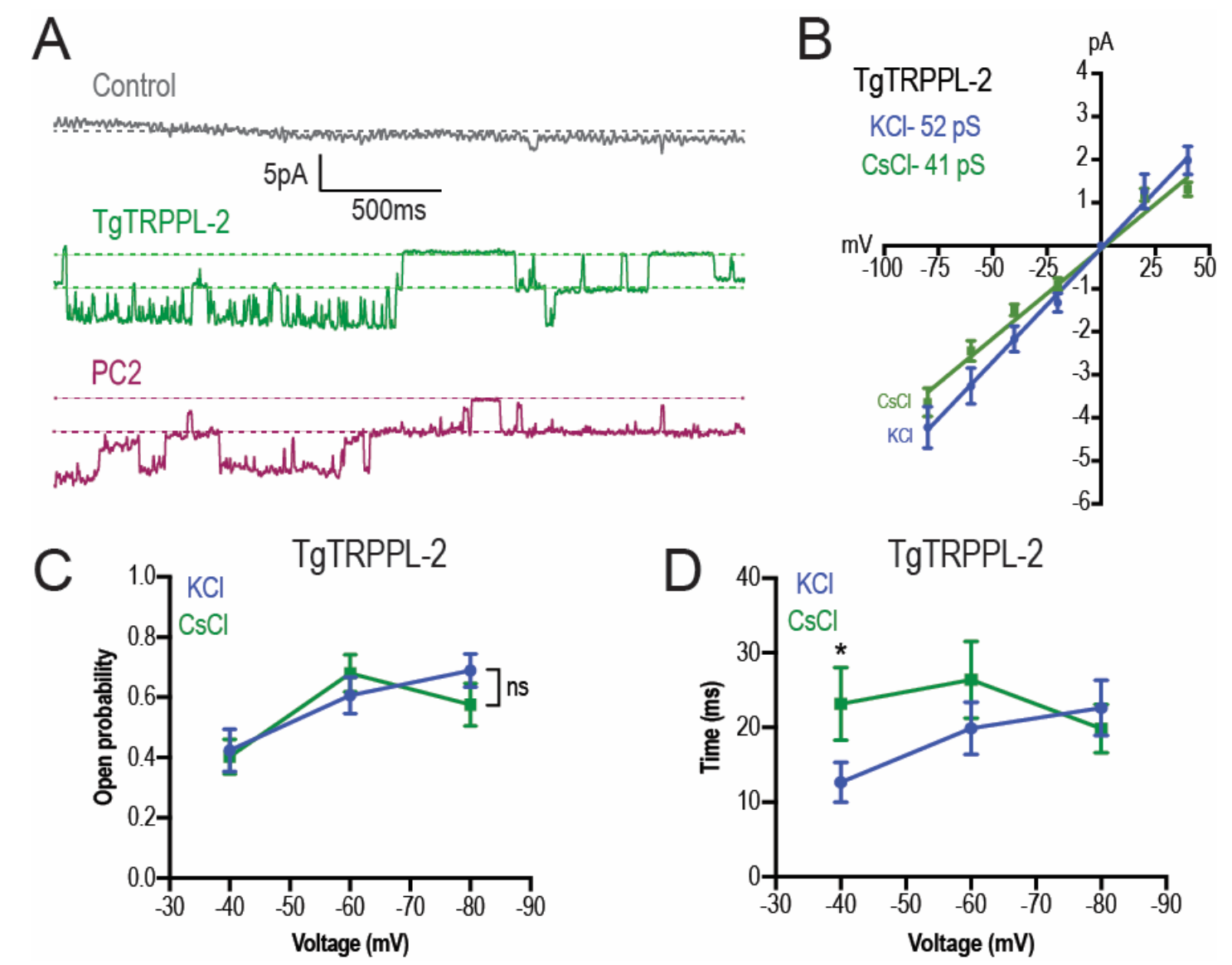

Figure 5. TgTRPPL-2 permeates $\mathbf{C a}^{2+}$. A. Representative tracing of currents recorded at $-80 \mathrm{mV}$ in the presence of $1.8 \mathrm{mM} \mathrm{Ca}^{2+}$ inside the pipette (Solution D, Table S4) of nuclear extracts from Control, TRPPL2- or PC2- expressing cells. Traces are a representation of 2 seconds of sampling from a total time of 25 seconds. B. Current-voltage relationship comparing single-channel conductance of TgTRPPL-2 cells in 1.8 $\mathrm{mM}$ in $\mathrm{KCl}$ (blue) or $\mathrm{CsCl}$ (green) buffer. Inset, Channel conductance of TgTRPPL-2 in the different conditions analyzed. C. Calculated open probability of TgTRPPL-2 expressing cells in the presence of 1.8 $\mathrm{mM} \mathrm{Ca}^{2+}$ in a $\mathrm{KCl}$ (blue) or $\mathrm{CsCl}$ (green) buffer. D. Average time of channel opening of TgTRPPL-2 expressing cells in the presence of $1.8 \mathrm{mM} \mathrm{Ca}^{2+}$ in a $\mathrm{KCl}$ (blue) or $\mathrm{CsCl}$ (green). ${ }^{*} p<0.04$. Values are means \pm SEM, 

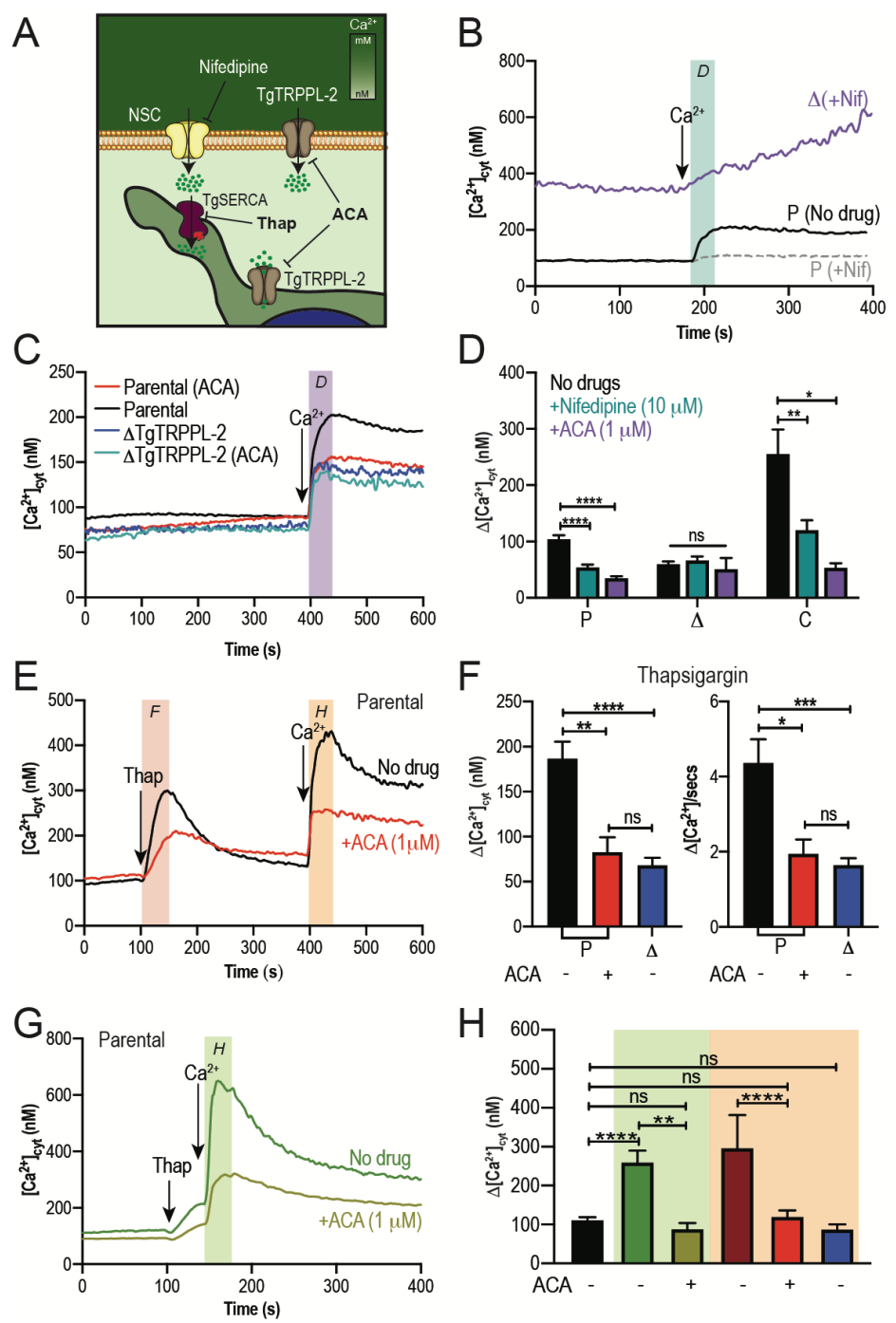

Figure 6. Regulation of TgTRPPL-2 by $\mathrm{Ca}^{2+}$ and inhibition by TRP inhibitors. A. Scheme showing TgTRPPL-2 at the PM and ER. B. Cytosolic $\mathrm{Ca}^{2+}$ measurements of Fura2 loaded tachyzoites pre-incubated with $10 \mu \mathrm{M}$ nifedipine. $1.8 \mathrm{mM} \mathrm{Ca}^{2+}$ was added where indicated. The blue box indicates the area used for the quantification presented in D. C. Cytosolic $\mathrm{Ca}^{2+}$ measurements of suspensions of parental and $\triangle T g T R P P L-2$ parasites pre-incubated for $3 \mathrm{~min}$ with ACA $(1 \mu \mathrm{M}) .1 .8 \mathrm{mM} \mathrm{Ca}^{2+}$ was added where indicated. The purple box shows the area used for the quantifications presented in D. D. Change in cytosolic $\mathrm{Ca}^{2+}$ during the first $20 \mathrm{~s}$ after addition of $\mathrm{Ca}^{2+}$ in the presence of $10 \mu \mathrm{M}$ of nifedipine or $1 \mu \mathrm{M}$ ACA. $* \mathrm{p}<0.01, * * \mathrm{p}<0.003, * * * * \mathrm{p}<0.0001$. E. Cytosolic $\mathrm{Ca}^{2+}$ increase after adding Thap $(1 \mu \mathrm{M})$ to a suspension of tachyzoites. The red line shows a similar experiment but the cells were pre-incubated with ACA for $3 \mathrm{~min}$. The pink and orange boxes show the areas used for the quantifications presented in F. F. Quantification and statistical analysis of the $\triangle \mathrm{Ca}^{2+}$ and slope $50 \mathrm{~s}$ after the addition of Thap in the presence or absence of ACA in parental and the $\triangle T g T R P P L-2$ mutant. $p<0.01$, ** $p$ $<0.003 * * * p<0.0003$. G. Stimulation of $\mathrm{Ca}^{2+}$ influx by pre-addition of thap in the presence or absence of $1 \mu \mathrm{M}$ ACA. The green box shows the area used for the quantifications presented in H. H. Quantification of change of cytosolic $\mathrm{Ca}^{2+} 20 \mathrm{~s}$ after the addition of $1.8 \mathrm{mM} \mathrm{Ca}^{2+}$ following the addition of Thap under different conditions. ** $p<0.001$, **** $p<0.00001$. The statistical analysis for all experiments were done from at least three independent trials using student's t-test. Values are means \pm SEM. 

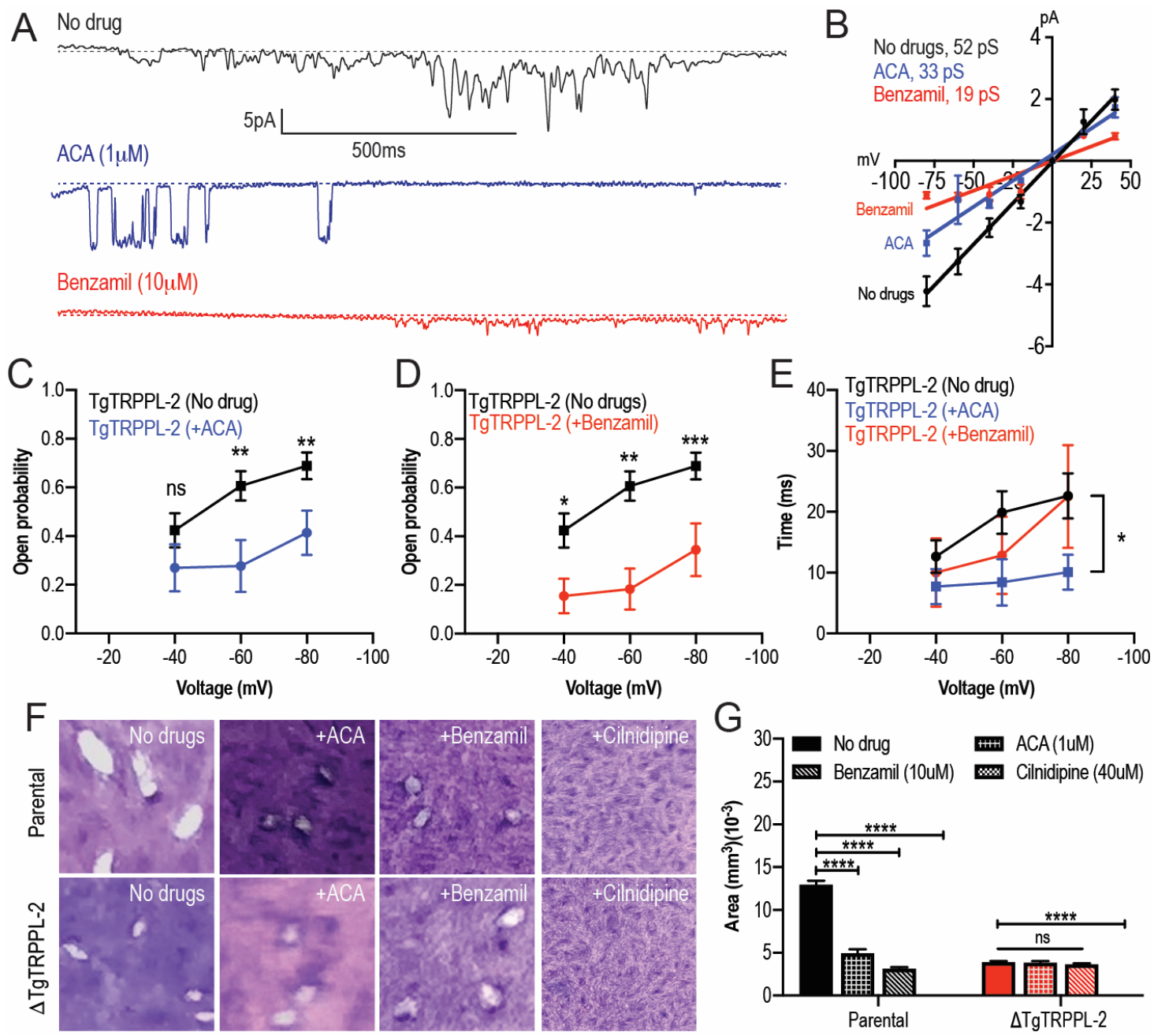

Figure 7. TRP Inhibitors decreased the activity of TgTRPPL-2. A. Example of currents recorded of TgTRPPL-2 expressing cells at $-80 \mathrm{mV}$ without inhibitors (Black trace) compared with the currents in the presence of $1 \mu \mathrm{M}$ of ACA (Blue trace) or $10 \mu \mathrm{M}$ of benzamil (Red trace). B. Current-voltage relationship comparing single-channel conductance in the presence of ACA or benzamil. The analysis was done from three independent biological trials and values are means \pm SEM. Inset, conductance of TgTRPPL-2 in the different conditions analyzed. C. Calculated open probability of TgTRPPL-2 expressing cells (black) or in the presence of ACA (blue). ${ }^{* *} p<0.006-0.007$. D. Calculated open probability of TgTRPPL-2 expressing cells (black) or in the presence of Benzamil (red). Asterisks indicate $\mathrm{p}$ values for significance. $* p<0.02$, $* * \mathrm{p}<0.002, * * * \mathrm{p}<0.0002$. E. Average time of channel opening of TgTRPPL-2 expressing cells in the presence of TRP inhibitors. Asterisks indicate $\mathrm{p}$ values for significance, $* p<0.02 \mathbf{F}$. Plaque assay of the $\triangle T g T R P P L-2$ mutant and the parental strain in the presence of ACA $(1 \mu \mathrm{M})$, benzamil $(10 \mu \mathrm{M})$ and cilnidipine $(40 \mu \mathrm{M})$ after 7 days of growth. G. Statistical analysis of plaque sizes done from three independent biological replicates using student's $t$-test. Values are means $\pm \mathrm{SEM}$, $* * * * p<0.0001$. 


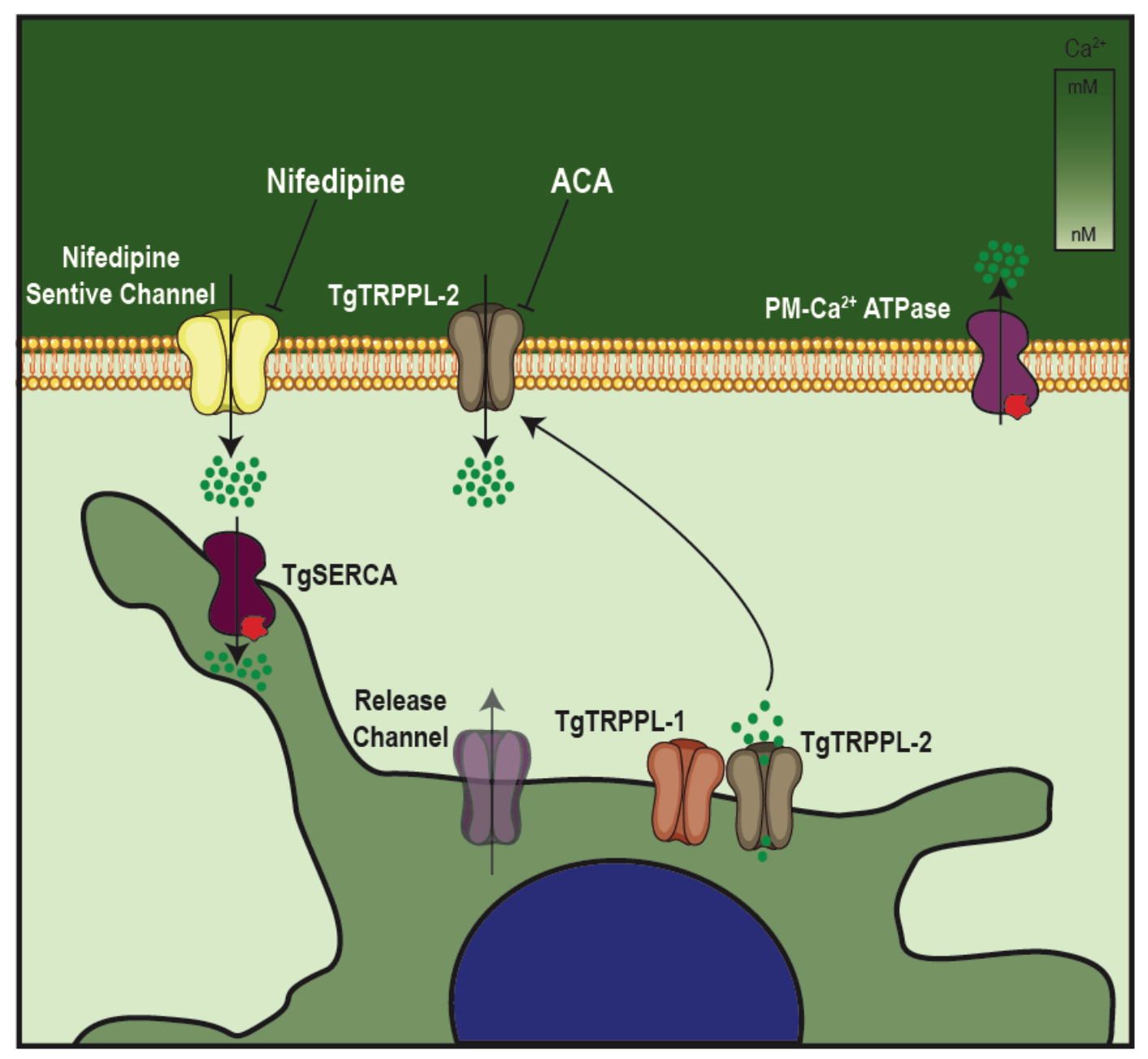

Figure 8. The role of TgTRPPL-2 in $\mathrm{Ca}^{2+}$ influx into the cytosol of $\mathrm{T}$. gondii. Schematic representation of $\mathrm{Ca}^{2+}$ influx into the cytosol of $T$. gondii. $\mathrm{Ca}^{2+}$ influx is mediated by two independent $\mathrm{Ca}^{2+}$ channels at the PM, a nifedipine-sensitive channel and TgTRPPL-2. TgTRPPL-2 localizes to the plasma membrane as well as the ER. TgTRPPL-2 at the PM is a cation permeable channel that mediates $\mathrm{Ca}^{2+}$ influx by a pathway that is activated by high $\left[\mathrm{Ca}^{2+}\right]$ and can be inhibited by broad TRP inhibitors like ACA and benzamil. The presence of the channel at the ER suggest that it may function as a $\mathrm{Ca}^{2+}$ efflux channel. Increase in cytosolic $\left[\mathrm{Ca}^{2+}\right]$ can modulate TgTRPPL-2 by allowing the channel to open for longer time thus allowing more $\mathrm{Ca}^{2+}$ to enter the cell. 